\title{
On a New Functional Equation for Local Integrals
}

\author{
Erez Lapid
}

Zhengyu Mao

Vienna, Preprint ESI 2404 (2012)

January 7, 2013

Supported by the Austrian Federal Ministry of Education, Science and Culture Available online at http://www.esi.ac.at 


\section{On a new functional equation for local integrals}

Erez Lapid and Zhengyu Mao

\section{CONTENTs}

1. Introduction and statement of main result 1

2. Basic estimates 8

3. Model transition - first case 14

4. Model transition - second case 21

5. The functional equation 28

References $\quad 30$

\section{Introduction and statement of main result}

Global integrals of Rankin-Selberg type are ubiquitous in the theory of automorphic forms and their $L$-functions and have been studied extensively in the literature. The prototype is the original construction for $\mathrm{GL}_{2} \times \mathrm{GL}_{2}\left[\right.$ Ran39, Sel40]. Its extension to $\mathrm{GL}_{m} \times \mathrm{GL}_{n}$ was the subject matter of intensive work by Jacquet, Piatetski-Shapiro and Shalika around 1980 [JPSS79a, JPSS79b, JS81b, JS81a] (with some parts completed only in recent years by Jacquet [Jac04, Jac09]).

Along with the global integrals there is a rich local theory (see [JPSS83, JS90] and the references above). The local zeta integrals admit functional equations which give rise to the local $L$ and $\epsilon$ factors. The purpose of this paper is to derive a variant of the local functional

2010 Mathematics Subject Classification. Primary 11F70.

Authors partially supported by U.S.-Israel Binational Science Foundation Grant \# 057/2008.

Second named author partially supported by NSF grant DMS 1000636 and by a fellowship from the Simons Foundation. 
equations of Rankin-Selberg integrals. The precise statement is Theorem 1.2 below. On a formal level, one can view these functional equations as a consequence of the ordinary ones. However, they differ in that they don't have mysterious proportionality constants. At any rate we will give a direct proof which is independent of the detailed local theory of Rankin-Selberg integrals. In the supercuspidal case this was proved in $[\mathbf{L M}]$. Here we prove the general case which is complicated by analytic difficulties.

The new functional equations admit a non-split analogue, which is also covered in Theorem 1.2. They are proved similarly, except that they depend on a certain growth condition which we do not address in this paper, but is probably within reach. Thus, our results in the non-split case are conditional.

The new functional equation is one of the ingredients of the paper $[\mathbf{L M}]$. In that paper we formulate a conjecture about Whittaker coefficients of classical groups and the metaplectic double cover of the symplectic group. For the latter, we reduce the global conjecture to a local one, and prove the local conjecture in the supercuspidal case. In a subsequent work we will use the results of this paper (among other things) to extend the argument of $[\mathbf{L M}]$ to the non supercuspidal case.

Rankin-Selberg integrals have been a favorite topic of PiatetskiShapiro. We hope that this note serves as a tribute to PiatetskiShapiro's legacy.

Acknolwedgement. We would like to thank Hervé Jacquet and Yiannis Sakellaridis for helpful discussions.

1.1. General notation. Let $F$ be a local field of characteristic 0 (although this is probably not essential) with normalized absolute value $|\cdot|$. In the non-archimedean case let $q$ be the size of the residue field.

In general we denote by bold face letters algebraic varieties over $F$ and by plain letters the set of $F$-points. Thus, $X=\mathbf{X}(F)$.

Let $E / F$ be a quadratic étale algebra over $F$ (either split or inert). Let $\mathbf{G}$ be the restriction of scalars of $\mathrm{GL}_{n}$ from $E$ to $F$. Thus $G=$ $\mathrm{GL}_{n}(E)$. Let $\tilde{\mathbf{G}}=\mathrm{GL}_{n}$ considered as a subgroup of $\mathbf{G}$. For any subgroup $\mathbf{X}$ of $\mathbf{G}$ we write $\tilde{\mathbf{X}}:=\mathbf{X} \cap \tilde{\mathbf{G}}$ (and correspondingly, $\tilde{X}=$ $X \cap \tilde{G})$.

Let $K$ be the standard maximal compact subgroup of $G$. We denote by $I_{m}$ the identity matrix in $\mathrm{GL}_{m}$ and by $g^{t}$ the transpose of the matrix $g$. 
Let $\mathbf{N}$ be the subgroup of upper unitriangular matrices in $\mathbf{G}$. Fix a unitary character $\psi: F \backslash E \rightarrow \mathbb{C}^{*}$. Let $\psi_{N}$ be the character of $N$, which is trivial on $\tilde{N}$, given by

$$
\psi_{N}(u)=\psi\left(u_{1,2}+\ldots+u_{n-1, n}\right), \quad u \in N .
$$

When there is no source of confusion we will often write $\psi$ instead of $\psi_{N}$.

By a representation of $G$ we will always mean either a smooth complex representation of finite length in the $p$-adic case or a smooth Fréchet representation of finite length and moderate growth in the archimedean case.

Let $\Pi(G)$ be the set of irreducible representations of $G, \Pi_{\text {gen }}(G)$ the subset of generic representations, and $\Pi_{\text {temp }}(G)$ the subset of tempered representations. For any $\pi \in \Pi_{\text {gen }}(G)$ we let $\mathcal{W}(\pi, \psi)$ be its Whittaker model: every $W \in \mathcal{W}(\pi, \psi)$ is a function satisfying

$$
W(u g)=\psi(u) W(g), \quad u \in N, g \in G
$$

which is of moderate growth on $N \backslash G$.

Recall that $\pi \in \Pi(G)$ is called $\tilde{G}$-distinguished if it admits a nontrivial continuous linear form $L$ on $\pi$ such that $L(\pi(g) v)=L(v)$ for all $v \in \pi$ and all $g \in \tilde{G}$. Recall that such $L$, if exists, is unique up to scalar. (This is clear in the split case; in the inert case see [Fli91, AG09].)

For $i=0, \ldots, n$ let $H_{i} \cong \mathrm{GL}_{n-i}(E)$ be the subgroup of $G$ consisting of $\left(\begin{array}{ll}h & \\ { } I_{i}\end{array}\right)$ where $h \in \mathrm{GL}_{n-i}(E)$ and let $N_{i}$ be the subgroup of upper unitriangular matrices in $H_{i}$. We also let $R_{i}$ be the unipotent subgroup of $G$ consisting of the matrices of the form $\left(\begin{array}{cc}I_{n-i} & \\ X & I_{i}\end{array}\right)$ where the last row of $X$ is zero.

Let $w_{m}$ be the permutation matrix corresponding to the longest Weyl element in $\mathrm{GL}_{m}$. Also for $i=0, \ldots, n$ let $w_{n, i}=\left({ }_{w_{n-i}} I_{i}\right)$. For $g \in G$, let $g^{\sigma}=\left(g^{t}\right)^{-1}$ - the Cartan involution, and $g^{*}=w_{n} g^{\sigma} w_{n}-$ the principal involution. For a subgroup $H$ of $G$ we denote by $H^{*}$ its image under *.

1.2. Let $\pi \in \Pi_{\text {gen }}(G)$. For $W \in \mathcal{W}(\pi, \psi), r=1, \ldots, n-1$, we consider the integrals

$$
\begin{gathered}
A_{r}(W, s)=\int_{\tilde{N}_{r} \backslash \tilde{H}_{r}} W(h)|\operatorname{det} h|^{s+1-r} d h, \\
A_{r}^{\prime}(W, s)=\int_{R_{r}} \int_{\tilde{N}_{r} \backslash \tilde{H}_{r}} W(h x)|\operatorname{det} h|^{s+1-r} d h d x,
\end{gathered}
$$


and

$$
B_{r}(W, s)=\int_{R_{r}^{*}} \int_{\left(\tilde{N}_{r}\right)^{*} \backslash\left(\tilde{H}_{r}\right)^{*}} W\left(h x w_{n, r}\right)|\operatorname{det} h|^{-(s+1-r)} d h d x .
$$

Since $W^{\sharp}(g):=W\left(w_{n} g^{\sigma}\right)=W\left(g^{*} w_{n}\right)$ is a Whittaker function (with respect to $\psi^{-1}$ ) in the space of the contragredient $\hat{\pi}$ of $\pi$, we can relate $A_{r}^{\prime}$ to $B_{r}$ as follows:

$$
B_{r}\left(W^{\sharp}, s\right)=A_{r}^{\prime}\left(\pi\left(w_{n, r}^{*} w_{n}\right) W, s\right) .
$$

The following Lemma is standard. For completeness we will prove it in $\S 2.2$ below.

Lemma 1.1. Let $\pi \in \Pi_{\text {gen }}(G)$ and $r=1, \ldots, n-1$. Then the integrals defining $A_{r}(W, s), A_{r}^{\prime}(W, s)$ and $B_{r}(W, s)$ converge absolutely for $\operatorname{Re} s \gg 0$ and these functions admit meromorphic continuation to $\mathbb{C}$. In the $p$-adic case they are rational functions in $q^{s}$. Moreover, if $\pi$ is tempered (resp., unitarizable) then $A_{r}(W, s)$ and $B_{r}(W, s), A_{r}^{\prime}(W, s)$ are absolutely convergent for $\operatorname{Re} s>-1$ (resp. Re $s \geq 0)$.

We remark that for $\pi$ unitarizable, the convergence of $A_{1}$ was proved in $[\mathbf{J S} 81 \mathbf{b}, \S 1,3]$ for the split case and in $[\mathbf{F l i 8 8}, \S 4]$ for the non-archimedean inert case.

For simplicity we write $A_{r}(W)=A_{r}(W, 0)$, and similarly for $B_{r}(W)$.

Our main result is

TheOREM 1.2. Let $\pi \in \Pi_{\text {gen }}(G)$ be unitararizable and $\tilde{G}$-distinguished and let $r=1, \ldots, n-1$. Then the identity

$$
A_{r}(W)=B_{r}(W), \quad W \in \mathcal{W}(\pi, \psi)
$$

holds provided that either

(1) $E / F$ is split,

(2) E/F is inert non-archimedean and Assumption 2.8 below holds, or,

(3) $E / F=\mathbb{C} / \mathbb{R}$ and Assumptions 2.8 and 2.10 below hold.

Note that the statement of Theorem 1.2 depends implicitly on the choice of Haar measures. If for any subgroup $X$ of $G$ the Haar measure on $X^{*}$ is compatible with that of $X$ then we only need to specify the Haar measure on $R_{r}$. To that end we will take the self-dual Haar measure on $E$ with respect to $\psi$ and use the identification $R_{i} \simeq E^{(r-1)(n-r)}$ (by coordinates).

REMARK 1.3. It is known that for any $\pi \in \Pi_{\text {gen }}(G), A_{1}(W, s)$ is holomorphic at $s=0$ (cf. [FLO12, appendix A]). Thus, $A_{1}(W)$ is defined. It is likely that the same is true for $A_{r}(W, s)$ (and $B_{r}(W, s)$ ) for 
$r>1$ and that Theorem 1.2 holds for any $\tilde{G}$-distinguished $\pi \in \Pi_{\text {gen }}(G)$. However, we will not discuss this issue here.

1.3. Let us give a purely formal argument for Theorem 1.2 in the split case (ignoring convergence issues). Write $\psi(a, b)=\psi_{1}(a-b)$ where $\psi_{1}$ is a non-trivial character of $F$. Also write $\pi=\pi_{1} \otimes \pi_{2}$ with $\pi_{2}=\hat{\pi}_{1}$. We can view $A_{r}\left(W_{1} \otimes W_{2}\right)$ as the inner product in $X=N_{r}(F) \backslash \mathrm{GL}_{n-r}(F)$ of $W_{1}|\operatorname{det}|^{(1-r) / 2}$ and $W_{2}|\operatorname{det}|^{(1-r) / 2}$. Expanding it spectrally we get

$$
\begin{aligned}
\int_{\Pi_{\text {gen }}\left(\mathrm{GL}_{n-r}(F)\right)} \sum_{W^{\prime}} \int_{X} W_{1}\left(\left({ }^{g}{ }_{I_{r}}\right)\right) W^{\prime}(g)|\operatorname{det} g|^{(1-r) / 2} d g \times \\
\int_{X} W_{2}\left(\left({ }^{g} I_{r}\right)\right) \overline{W^{\prime}(g)}|\operatorname{det} g|^{(1-r) / 2} d g d_{\mu} \sigma
\end{aligned}
$$

for an appropriate spectral measure $d_{\mu}$ where $W^{\prime}$ range over an orthonormal basis in the $\psi_{1}^{-1}$-Whittaker space of $\sigma$. The usual functional equation in Rankin-Selberg theory (e.g. [JPSS83]) gives a relation (in the sense of analytic continuation)

$$
\begin{aligned}
\int_{X} \int_{R_{r}^{*}} W_{1}\left(\left(\begin{array}{ll}
I_{r} & g
\end{array}\right) x w_{n, r}\right) W^{\prime}(g)|\operatorname{det} g|^{r / 2+s-1} d x d g \\
\quad= \pm \gamma\left(s, \pi_{1} \times \sigma, \psi_{1}\right) \int_{X} W_{1}\left(\left(\begin{array}{cc}
g & I_{r}
\end{array}\right)\right) W^{\prime}(g)|\operatorname{det} g|^{s-r / 2} d g .
\end{aligned}
$$

In particular, for $s=\frac{1}{2}$ we get

$$
\begin{aligned}
\int_{X} \int_{R_{r}^{*}} W_{1} & \left(\left(\begin{array}{ll}
I_{r} & g
\end{array}\right) x w_{n, r}\right) W^{\prime}(g)|\operatorname{det} g|^{(r-1) / 2} d x d g \\
= & \pm \gamma\left(\frac{1}{2}, \pi_{1} \times \sigma, \psi_{1}\right) \int_{X} W_{1}\left(\left({ }^{g} I_{r}\right)\right) W^{\prime}(g)|\operatorname{det} g|^{(1-r) / 2} d g
\end{aligned}
$$

Similarly,

$$
\begin{aligned}
\int_{X} \int_{R_{r}^{*}} W_{2}\left(\left({ }^{I_{r}} g\right) x w_{n, r}\right) \overline{W^{\prime}(g)}|\operatorname{det} g|^{(r-1) / 2} d x d g \\
= \pm \gamma\left(\frac{1}{2}, \pi_{2} \times \hat{\sigma}, \psi_{1}^{-1}\right) \int_{X} W_{2}\left(\left({ }^{g} I_{r}\right)\right) \overline{W^{\prime}(g)}|\operatorname{det} g|^{(1-r) / 2} d g
\end{aligned}
$$

Since

$$
\gamma\left(\frac{1}{2}, \pi_{1} \times \sigma, \psi_{1}\right) \gamma\left(\frac{1}{2}, \pi_{2} \times \hat{\sigma}, \psi_{1}^{-1}\right)=1
$$


$A_{r}\left(W_{1} \otimes W_{2}\right)$ equals

$$
\begin{array}{r}
\int_{\Pi_{\mathrm{gen}}\left(\mathrm{GL}_{n-r}(F)\right)} \sum_{W^{\prime}} \int_{X} \int_{R_{r}^{*}} W_{1}\left(\left({ }^{I_{r}} g\right) x w_{n, r}\right) W^{\prime}(g)|\operatorname{det} g|^{(r-1) / 2} d x d g \\
\times \int_{X} \int_{R_{r}^{*}} W_{2}\left(\left(\begin{array}{l}
I_{r} \\
g
\end{array}\right) x w_{n, r}\right) \overline{W^{\prime}(g)}|\operatorname{det} g|^{(r-1) / 2} d x d g d_{\mu} \sigma
\end{array}
$$

Once again, this is the spectral expansion of $B_{r}\left(W_{1} \otimes W_{2}\right)$.

The rigorous proof of Theorem 1.2 will be given in the following sections. The idea of the proof is to express $A_{r}$ as a Fourier coefficient of $A_{1}$ with respect to the unipotent radical $U_{r+1}$ of the standard parabolic subgroup of type $(n-r+1,1, \ldots, 1)$. (We call this procedure "model transition".) Similarly, we express $A_{r}^{\prime}$ as a Fourier coefficient of $A_{1}^{\prime}=$ $A_{1}$ with respect to a conjugate of $U_{r+1}^{*}$. This is done in $\S 3$ and $\S 4$ respectively. Finally, we use the relation (14) below to get the required identity. The definitions of the Fourier coefficients above require some care since they are not given by absolutely convergent integrals. The analysis will be facilitated by the estimates of $\S 2$.

1.4. Let us introduce some more notation which will be used for the rest of the paper.

Let $\mathbf{T}$ be the group of diagonal matrices in $\mathbf{G}$. We write an element $t \in T$ as $\operatorname{diag}\left(t_{1}, \ldots, t_{n}\right)$. Let $\mathbf{N}^{\circ}$ be the derived group of $\mathbf{N}$. For any subgroup $\mathbf{X}$ of $\mathbf{N}$ we write $\mathbf{X}^{\circ}=\mathbf{X} \cap \mathbf{N}^{\circ}$ which is a normal subgroup of $\mathbf{X}$ containing (possibly strictly) the derived group of $\mathbf{X}$; let $\mathbf{X}^{\mathrm{ab}}=$ $\mathbf{X}^{\circ} \backslash \mathbf{X}$. Let $\mathcal{P}$ be the mirabolic subgroup of $G$ consisting of elements whose last row is $(0, \ldots, 0,1)$.

For $i=1, \ldots, n-1$, we introduce some more algebraic subgroups of $G$.

- Let $G_{i} \cong \mathrm{GL}_{i}(E)$ be the subgroup of $G$ consisting of $\left({ }^{I_{n-i}}{ }_{h}\right)$ where $h \in \mathrm{GL}_{i}(E)$.

- Let $V_{i}$ be the unipotent radical of the standard parabolic subgroup of $G$ of type $(n-i, i)$.

- Let $U_{i}$ be the unipotent radical of the standard parabolic subgroup of $G$ of type $(n-i, 1, \ldots, 1)$.

- Let $C_{i}=U_{i} \cap H_{i-1}$, consisting of elements $u=I_{n}+v$ in $N$ where $v$ is zero outside the $(n-i+1)$-th column. Then $U_{i}=C_{i} \ltimes U_{i-1}$ (with $U_{0}=1$ ).

- For $k=1, \ldots, i$ let $C_{i, k}=C_{k} \cap V_{i}$ and ${ }_{k} C_{i}=C_{k} \cap G_{i}$.

- Let $D_{i}=C_{i+1}^{t}, D_{i, k}=D_{k} \cap V_{i}^{t}$ and ${ }_{k} D_{i}=D_{k} \cap G_{i}$. Thus $R_{i}=\prod_{k=1}^{i-1} D_{i, k}$. 
- Let $T_{i}$ be the $i$-dimensional torus

$$
T_{i}=T \cap G_{i+1} \cap \mathcal{P}=\left\{\operatorname{diag}\left(t_{1}, \ldots, t_{n}\right): t_{1}=\cdots=t_{n-i-1}=t_{n}=1\right\} .
$$

- For $m=1, \ldots, r$ let $R_{r, m}=R_{r} \cap H_{m}=\prod_{k=m}^{r-1} D_{r, k}$. In particular, $R_{r, 1}=R_{r}$ and $R_{r, r}=1$.

- Let $\alpha_{i}$ be the simple root $\alpha_{i}(t)=\frac{t_{i}}{t_{i+1}}$ of $T$ and let $N_{\alpha_{i}}$ denote the corresponding one parameter root subgroup of $N$. It is the image of the homomorphism $\lambda_{i}: E \rightarrow N$ given by $\lambda_{i}(x)=$ $I_{n}+x e_{i, i+1}, x \in E$ where $e_{i, j}$ is the matrix whose $(i, j)$-th entry is 1 and all other entries are zero.

For a group $\mathbf{X}$ defined over $F$, we denote the quotient $\mathbf{X}(F) \backslash \mathbf{X}(E)$ by $[X]$. For example $\left[U_{i}^{\mathrm{ab}}\right]=\left(\tilde{U}_{i}^{\circ} \backslash \tilde{U}_{i}\right) \backslash\left(U_{i}^{\circ} \backslash U_{i}\right)$ is isomorphic to $\prod_{k=n-1}^{n-i}\left[N_{\alpha_{k}}\right]$ through the embedding of $N_{\alpha_{k}}$ in $U_{i}$. If $X \subset N$, we can consider $\psi$ as a character on $\left[X^{\mathrm{ab}}\right]$.

If $f$ is a function on a group $Y$ and $X=Y \ltimes Z$, then we often extend $f$ to a function on $X$ via the quotient map $X \rightarrow Y$.

For any group $X$ we write $\delta_{X}$ for the modulus function of $X$. We also write $\delta_{0}$ for the modulus function of the upper triangular Borel subgroup $T \ltimes N$. Similarly for $\tilde{\delta}_{0}$.

If $X$ is a locally compact Abelian group then we denote by $\widehat{X}$ its Pontryagin dual.

Note that $\left[C_{i}^{\circ}, D_{i}\right]=N_{\alpha_{n-i}}$ where as usual we denote by $[x, y]$ the commutator $x y x^{-1} y^{-1}$. This gives rise to a non-degenerate pairing between $C_{i}^{\circ}$ and $D_{i}$ given by $\langle c, d\rangle=\langle c, d\rangle_{\psi}=\psi([c, d])^{-1}$. Moreover, $\langle\cdot, \cdot\rangle$ induces non-degenerate pairings between $\left[C_{i}^{\circ}\right]$ and $\tilde{D}_{i}$, between $C_{m, i}$ and $D_{m, i}$, and between ${ }_{i} C_{m}^{\circ}$ and ${ }_{i} D_{m}$ for $i<m<n$.

For any Whittaker function $W$ on $G$ with respect to $\psi$ we have the following basic property:

$$
W(h z x y)=\psi(z)\langle y, x\rangle W(h x), \quad h \in H_{i+1}, x \in D_{i}, y \in C_{i}^{\circ}, z \in C_{i} .
$$

The convention of Haar measures will be as follows. Recall that we fixed the Haar measure on $E$ to be the self-dual Haar measure with respect to $\psi$. We also fix once and for all an arbitrary Haar measure on $F$. Note that $\psi$ gives rise to an isomorphism between $\widehat{E / F}$ and $F$. Under this isomorphism, the Haar measure on $\widehat{E / F}$ dual to the quotient measure on $E / F$ coincides with the Haar measure on $F$.

In the sequel we will encounter Haar measures on two types of algebraic subgroups of $G$ (or quotients thereof): subgroups of $\tilde{G}$ and unipotent subgroups of $G$. In the former case, we will take the unnormalized Tamagawa measure with respect to the "obvious" gauge form 
and the above measure on $F$. For instance for $\tilde{G}$ we take the gauge form $\frac{\wedge d g_{i, j}}{\operatorname{det} g^{n}}$; for unipotent groups we take the product of the differentials of the coordinates; for diagonal groups we take the logarithmic differentials of the coordinates. In the second case, all unipotent subgroups of $G$ in hand will have natural coordinates and we will take the product of the Haar measures of the coordinates (using the above measure on $E)$.

We endow all compact groups with Haar measures of total volume one.

We will use the following convention for iterated and double integrals: we write

$$
\int_{A}\left(\int_{B} \int_{C} f(a, b, c) d c d b\right) d a
$$

to mean that the double integral over $B$ and $C$ converges and the integral over $A$ (as an iterated integral) converges.

\section{Basic estimates}

2.1. For any $\pi \in \Pi_{\text {gen }}(\tilde{G})$ and $s \in \mathbb{R}$ we write $\pi[s]=\pi \otimes|\operatorname{det} \cdot|^{s}$. Let $\pi \in \Pi_{\text {gen }}(\tilde{G})$. Denoting by $\times$ parabolic induction. We write

$$
\pi=\pi_{1}\left[s_{1}\right] \times \cdots \times \pi_{k}\left[s_{k}\right]
$$

with $\pi_{i}$ square-integrable and $s_{1}, \ldots, s_{k} \in \mathbb{R}$. This presentation is unique up to permutation. By an abuse of language we call the $s_{i}$ 's the exponents of $\pi$. For instance, if $\pi$ is tempered then the exponents are 0 . If $\pi$ is unitarizable then the exponents are in the open interval $\left(-\frac{1}{2}, \frac{1}{2}\right)$.

We will need the following variant of the estimates of [JPSS79a].

LEMMA 2.1. Suppose that $\pi \in \Pi_{\text {gen }}(\tilde{G})$ with unitary central character and all its exponents are bigger than $\lambda$. Then for any $W$ in the Whittaker space of $\pi$ there exists a Schwartz function $\phi \in \mathcal{S}\left(F^{n-1}\right)$ such that

$$
|W(t k)| \leq \tilde{\delta}_{0}^{\frac{1}{2}}(t)|\operatorname{det} t|^{\lambda}\left|t_{n}\right|^{-n \lambda} \phi\left(\alpha_{1}(t), \ldots, \alpha_{n-1}(t)\right)
$$

for all $t \in \tilde{T}, k \in \tilde{K}$.

Proof. Indeed, in the non-archimedean case, it is easy to see that for any standard parabolic $\tilde{P}=\tilde{M} \tilde{U}$, any cuspidal exponent of $\pi$ along $\tilde{P}$ (viewed as a $\mathbb{R}_{>0}$-valued quasi-character of the center $Z(\tilde{M})$ of $\tilde{M}$ ) has the form

$$
t \mapsto|\operatorname{det} t|^{\lambda}\left|t_{n}\right|^{-n \lambda} \prod_{i=1}^{n-1}\left|\alpha_{i}(t)\right|^{x_{i}}, \quad t=\operatorname{diag}\left(t_{1}, \ldots, t_{n}\right) \in Z(\tilde{M})
$$


with $x_{i} \geq 0$ for all $i$. The estimate (6) follows from [LM09, Theorem 3.1]. In the Archimedean case the result follows from Lemma 15.2.3 and statement (1) on the bottom of p. 369 of [Wal92]. We note that the $\Lambda$ in [ibid.] is of the form $\rho+n \lambda \varpi+\sum y_{i} \alpha_{i}$ where $y_{i} \geq 0$, $\rho$ is the half-sum of positive roots and $\varpi$ is the fundamental weight corresponding to $\alpha_{n-1}$.

Suppose that $E / F$ is inert. Then we define exponents for $\pi \in$ $\Pi_{\text {gen }}(G)$ in a similar way (with $|\cdot|_{E}$ instead of $\left.|\cdot|\right)$. If $E / F$ splits then the exponents of $\pi=\pi_{1} \otimes \pi_{2} \in \Pi_{\text {gen }}(G)$ are by definition the union of the exponents of $\pi_{i}, i=1,2$.

Corollary 2.2. Suppose that $\pi \in \Pi_{\text {gen }}(G)$ with unitary central character and all its exponents are bigger than $\lambda$. Then for any $W \in$ $\mathcal{W}(\pi, \psi)$ there exists a Schwartz function $\phi \in \mathcal{S}\left(F^{n-1}\right)$ such that

$$
|W(t k)| \leq \delta_{0}^{\frac{1}{2}}(t)|\operatorname{det} t|^{2 \lambda}\left|t_{n}\right|^{-2 n \lambda} \phi\left(\alpha_{1}(t), \ldots, \alpha_{n-1}(t)\right)
$$

for all $t \in \tilde{T}, k \in \tilde{K}$.

2.2. Next, we will prove the following slightly stronger version of Lemma 1.1. The argument is standard.

Lemma 2.3. Let $\pi \in \Pi_{\text {gen }}(G), W \in \mathcal{W}(\pi, \psi)$ and $r=1, \ldots, n-1$. Suppose that the exponents of $\pi$ are $\geq \lambda$. Then $A_{r}(W, s), A_{r}^{\prime}(W, s)$ and $B_{r}(W, s)$ are given by absolutely convergent integrals for $\operatorname{Re} s>-2 \lambda-1$ which extend to meromorphic functions in $s \in \mathbb{C}$. In the p-adic case they are rational functions in $q^{s}$. Moreover for any $s$ in the range of convergence there exists $N$ and $C$ such that

$$
\left|A_{r}(\pi(g) W, s)\right|,\left|A_{r}^{\prime}(\pi(g) W, s)\right| \leq C\|g\|^{N} .
$$

Proof. We start with $A_{r}$. Let $\tilde{K}_{r}$ be the standard maximal compact subgroup of $\tilde{H}_{r}, \tilde{T}_{r}^{\prime}$ the diagonal torus in $H_{r}$ and $\tilde{\delta}_{r}$ the modulus function of the upper triangular Borel subgroup $\tilde{T}_{r}^{\prime} \ltimes \tilde{N}_{r}$ of $\tilde{H}_{r}$. We rewrite $A_{r}$ using the Iwasawa decomposition as

$$
A_{r}(W, s)=c_{r} \int_{\tilde{K}_{r}} \int_{\tilde{T}_{r}^{\prime}} W(t k)|\operatorname{det} t|^{s+1-r} \tilde{\delta}_{r}^{-1}(t) d t d k
$$

for a suitable constant $c_{r}>0$. Note that $\delta_{0}^{\frac{1}{2}}(t) \tilde{\delta}_{r}^{-1}(t)=|\operatorname{det} t|^{r}$. Therefore by (7) there exists $\phi \in \mathcal{S}\left(F^{n-1}\right)$ such that

$$
|W(t k)||\operatorname{det} t|^{\operatorname{Re} s+1-r} \tilde{\delta}_{r}^{-1}(t) \leq|\operatorname{det} t|^{\operatorname{Re} s+1+2 \lambda} \phi\left(\alpha_{1}(t), \ldots, \alpha_{n-1}(t)\right)
$$

for all $t \in \tilde{T}_{r}^{\prime}, k \in \tilde{K}_{r}$. Thus the integral (8) converges provided that $\operatorname{Re} s>-2 \lambda-1$ as claimed. 
The meromorphic continuation of $A_{r}(W, s)$ also follows from (8) by using the asymptotic behavior of Whittaker functions (cf. [JPSS79a] for the $p$-adic case and [Jac04] for the archimedean case).

We turn to $A_{r}^{\prime}$. Again, we use Iwasawa decomposition to write it as

$$
c_{r} \int_{R_{r}} \int_{\tilde{K}_{r}} \int_{\tilde{T}_{r}^{\prime}} W(t x k)|\operatorname{det} t|^{s+1-r} \tilde{\delta}_{r}^{-1}(t) d t d k d x .
$$

We note that the Iwasawa decomposition of $x$ is given by $u a k^{\prime}$ where $a=\operatorname{diag}\left(a_{1}, \ldots, a_{n}\right)$ with $a_{n}=1$ and $\left|a_{n-r+1}\right|_{E} \geq \max \left|x_{i, j}\right|_{E}$ in the inert case and $\left|a_{n-r+1}^{(l)}\right| \geq \max \left|x_{i, j}^{(l)}\right|, l=1,2$ in the split case where we write $a_{i}=\left(a_{i}^{(1)}, a_{i}^{(2)}\right)$ and similarly for the $x_{i, j}$ 's. The convergence follows as in the case of $A_{r}$. (It will also follow from the argument below.)

The moderate growth property of $A_{r}$ and $A_{r}^{\prime}$ is also clear.

Let us prove meromorphic continuation of $A_{r}^{\prime}$. Recall that for $m=$ $1, \ldots, r$ we write $R_{r, m}=R_{r} \cap H_{m}$. Thus, $R_{r, 1}=R_{r}$ and $R_{r, r}=1$. Recall that $C_{r, m}$ and $D_{r, m}$ are in duality via the commutator. Observe that for $m<r$, by (5) (applied with $i=m, h=h y$ and $z=e$ ) for any $\phi \in \mathcal{S}\left(C_{r, m}\right)$ we have

$$
\pi(\phi) W(h y x)=\hat{\phi}(x) W(h y x)
$$

for all $h \in H_{r}, x \in D_{r, m}$ and $y \in R_{r, m+1}$.

We introduce auxiliary integrals

$$
A_{r, m}(W)=\int_{R_{r, m}} \int_{\tilde{N}_{r} \backslash \tilde{H}_{r}} W(h x)|\operatorname{det} h|^{s+1-r} d h d x .
$$

Using the Dixmier-Malliavin theorem and (9), one proves the meromorphic continuation of $A_{r, m}$ by descending induction on $m$. Since $A_{r}^{\prime}=A_{r, 1}$ the analytic properties of $A_{r}^{\prime}$ follow.

Finally, the case of $B_{r}$ follows from the relation (4).

REMARK 2.4. Note that the use of the Dixmier-Malliavin Theorem is not essential. It suffice to write $W$ as a convolution by a sufficiently smooth test function (which is elementary) in order to get the meromorphic continuation strip by strip. The same remark applies to all future applications of the Dixmier-Malliavin Theorem.

2.3. Next, we discuss the split case in more detail. For any $\lambda \in \mathbb{C}^{n}$ we write

$$
|t|^{\lambda}=\prod_{i=1}^{n}\left|t_{i}\right|^{\lambda_{i}}, \quad t=\operatorname{diag}\left(t_{1}, \ldots, t_{n}\right) \in \tilde{T} .
$$


For any $\lambda \in \mathbb{C}^{n}$ let $\varphi_{\lambda}$ be the function on $\tilde{G}$ given by

$$
\varphi_{\lambda}(n t k)=\tilde{\delta}_{0}^{\frac{1}{2}}(t)|t|^{\lambda}, \quad n \in \tilde{N}, t \in \tilde{T}, k \in \tilde{K} .
$$

Let $f_{0}$ be the function on $\tilde{N}^{\text {ab }}$ given by

$$
f_{0}(v)=\int_{\tilde{N}^{\circ}} \varphi_{0}\left(w_{n} u v\right) d u .
$$

For convenience let $\psi^{\prime}$ be a non-degenerate character of $\tilde{N}$. Recall the Jacquet integral

$$
J(g, \lambda)=\int_{\tilde{N}} \varphi_{\lambda}\left(w_{n} u g\right) \psi^{\prime}(u)^{-1} d u .
$$

The integral converges absolutely for $\operatorname{Re} \lambda_{1}>\cdots>\operatorname{Re} \lambda_{n}$ and admits holomorphic continuation to $\lambda \in \mathbb{C}^{n}$. Moreover, $J(g, \lambda)$ is a Whittaker function for the induced representation $I(\lambda)$. In particular, for any $\lambda \in \mathbb{C}^{n}$ such that $\operatorname{Re} \lambda$ is dominant there exists $\phi \in \mathcal{S}\left(F^{n-1}\right)$ such that

$$
|J(t, \lambda)| \leq \phi\left(\alpha_{1}(t), \ldots, \alpha_{n-1}(t)\right) \tilde{\delta}_{0}^{\frac{1}{2}}(t)|t|^{\operatorname{Re} w_{n} \lambda}
$$

for all $t \in \tilde{T}$.

LEMMA 2.5. $f_{0}$ is defined (i.e., the integral (10) converges) and $f_{0} \in L^{2}\left(\tilde{N}^{\mathrm{ab}}\right)$. Moreover, its Fourier transform at $\psi_{t}^{\prime}=\psi^{\prime}\left(t \cdot t^{-1}\right)^{-1}$, $t \in \tilde{T}$ is given by $\tilde{\delta}_{0}^{-\frac{1}{2}}(t) J(t, 0)$.

Proof. We follow the argument of [SV, Lemma 6.3.1]. For Re $\lambda$ dominant, the function

$$
f_{\lambda}(v)=\int_{\tilde{N}^{\circ}} \varphi_{\lambda}\left(w_{n} u v\right) d u, \quad v \in \tilde{N}^{\mathrm{ab}}
$$

is smooth and belongs to $L^{1}\left(\tilde{N}^{\mathrm{ab}}\right)$. Its Fourier transform at $\psi_{t}^{\prime}$ is $\tilde{\delta}_{0}^{-\frac{1}{2}}(t)|t|^{-w_{n} \lambda} J(t, \lambda)$. Note that $\left\{\psi_{t}^{\prime}: t \in \tilde{T}\right\}$ is a co-null subset of $\widetilde{\tilde{N}^{\mathrm{ab}}}$ and that the push forward of the measure $\left|t_{1}\right| d t$ on $\tilde{T}$ via $t \mapsto \psi_{t}^{\prime}$ is the restriction of the Haar measure on $\widehat{\tilde{N}^{\text {ab }}}$. Therefore, by Fourier inversion we have

$$
f_{\lambda}\left(I_{n}\right)=\int_{\tilde{T} \cap \tilde{\mathcal{P}}} J(t, \lambda)|t|^{-w_{n} \lambda} \tilde{\delta}_{0}^{-\frac{1}{2}}(t)\left|t_{1}\right| d t .
$$

(The integral on the right-hand side converges absolutely by (11).) Fix a dominant $\lambda_{0} \in \mathbb{R}^{n}$. We claim that for any $\epsilon>0$ there exists $\phi \in \mathcal{S}\left(F^{n-1}\right)$ such that

$$
|J(t, \lambda)| \tilde{\delta}_{0}^{-\frac{1}{2}}(t) \leq|t|^{\epsilon w_{n} \lambda_{0}} \phi\left(\alpha_{1}(t), \ldots, \alpha_{n-1}(t)\right)
$$


for all $t \in \tilde{T} \cap \tilde{\mathcal{P}}$ and $\lambda=s \lambda_{0}$ with $|s| \leq \epsilon$. Indeed, by the maximum modulus principle it is enough to prove this inequality for $|s|=\epsilon$. In the $p$-adic case this follows immediately from the fact that on the set of regular $\lambda$ we have

$$
J(t, \lambda) \tilde{\delta}_{0}^{-\frac{1}{2}}(t)=\mathbf{1}_{I^{n-1}}\left(\alpha_{1}(t), \ldots, \alpha_{n-1}(t)\right) \sum_{w \in S_{n}} c_{w}(\lambda)|t|^{w \lambda}
$$

where $I$ is the largest fractional ideal on which $\psi^{\prime}$ is trivial, $S_{n}$ is the Weyl group and $c_{w}$ are holomorphic functions on the set of regular $\lambda$ (which can be explicated by Shintani's formula [Shi76]). The Archimedean case follows from [Jac04, Theorem 2].

Take $\lambda=s \lambda_{0}, s \in(0,1)$. It follows from (13) and the dominated convergence theorem that the right-hand side of (12) is a continuous function at $s=0$. On the other hand $\lambda \mapsto \varphi_{\lambda}\left(w_{n} u\right)$ is monotone decreasing in $s$. Therefore by Lebesgue's monotone convergence theorem (or Fatou's lemma) we conclude the convergence of (10) for $v=I_{n}$. The same argument works for any $v \in \tilde{N}^{\text {ab }}$, or alternatively we can use the fact that $\varphi_{0}(g v) \leq \max _{k \in \tilde{K}} \varphi_{0}(k v) \varphi_{0}(g)$ for all $g \in \tilde{G}$.

Moreover, for Re $\lambda$ dominant we have

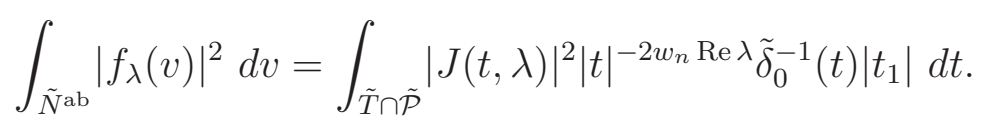

Once again, taking $\lambda=s \lambda_{0}$, the right-hand side is continuous at $s=0$, which implies that $f_{0} \in L^{2}\left(\tilde{N}^{\mathrm{ab}}\right)$. In addition, it follows once again from $(13)$ that $\widehat{f_{\lambda}}$ converges in $L^{2}\left(\widehat{\tilde{N}^{\text {ab }}}\right)$ as $s \rightarrow 0$. Hence, $f_{\lambda}$ converges (necessarily to $\left.f_{0}\right)$ in $L^{2}\left(\tilde{N}^{\text {ab }}\right)$ as $s \rightarrow 0$. We deduce that

$$
\widehat{f}_{0}=\lim _{s \rightarrow 0} \widehat{f}_{\lambda}
$$

in $L^{2}\left(\widehat{\tilde{N}^{\mathrm{ab}}}\right)$ and therefore $\widehat{f}_{0}\left(\psi_{t}^{\prime}\right)=\tilde{\delta}_{0}^{-\frac{1}{2}}(t) J(t, 0)$ for all $t \in \tilde{T}$ as required.

Let $\Xi$ be the standard zonal spherical function of $\tilde{G}$, i.e.

$$
\Xi(g)=\int_{\tilde{K}} \varphi_{0}(k g) d k=c \int_{\tilde{N}} \varphi_{0}\left(w_{n} u g\right) \varphi_{0}\left(w_{n} u\right) d u, \quad g \in \tilde{G}
$$

for some scalar $c>0$.

Lemma 2.6. The function $v \mapsto \int_{\tilde{N}^{\circ}} \Xi(u v) d u, v \in \tilde{N}^{\mathrm{ab}}$ is welldefined and belongs to $L^{2}\left(\tilde{N}^{\mathrm{ab}}\right)$. 
Proof. For any $v \in \tilde{N}^{\text {ab }}$ we have

$$
\begin{aligned}
c^{-1} \int_{\tilde{N}^{\circ}} \Xi(u v) d u & =\int_{\tilde{N}^{\circ}} \int_{\tilde{N}} \varphi_{0}\left(w_{n} v^{\prime} u v\right) \varphi_{0}\left(w_{n} v^{\prime}\right) d v^{\prime} d u \\
& =\int_{\tilde{N}^{\circ}} \int_{\tilde{N}} \varphi_{0}\left(w_{n} u v^{\prime} v\right) \varphi_{0}\left(w_{n} v^{\prime}\right) d v^{\prime} d u \\
& =\int_{\tilde{N}^{\mathrm{ab}}} \int_{\tilde{N}^{\circ}} \int_{\tilde{N}^{\circ}} \varphi_{0}\left(w_{n} u v_{1} v_{2} v\right) \varphi_{0}\left(w_{n} v_{1} v_{2}\right) d v_{1} d u d v_{2} \\
& =\int_{\tilde{N}^{\mathrm{ab}}} \int_{\tilde{N}^{\circ}} \int_{\tilde{N}^{\circ}} \varphi_{0}\left(w_{n} u v_{2} v\right) \varphi_{0}\left(w_{n} v_{1} v_{2}\right) d v_{1} d u d v_{2} \\
& =\int_{\tilde{N}^{\mathrm{ab}}} f_{0}\left(v^{\prime} v\right) f_{0}\left(v^{\prime}\right) d v^{\prime}<\infty
\end{aligned}
$$

by the previous lemma. Thus, also by the previous lemma, $v \mapsto$ $\int_{\tilde{N}^{\circ}} \Xi(u v) d u$ is the Fourier transform of $c|J(t, 0)|^{2} \tilde{\delta}_{0}^{-1}(t)$. Hence $\int_{\tilde{N}^{\circ}} \Xi(u \cdot) d u \in$ $L^{2}\left(\tilde{N}^{\mathrm{ab}}\right)$.

From Lemma 2.6 and the fact that smooth matrix coefficients of a tempered representation are bounded by $\Xi([\mathbf{C H H 8 8 , ~ S u n 0 9 ] )}$ we get

Corollary 2.7. Suppose that $E / F$ is split and $\pi \in \Pi_{\text {temp }}(G)$ is $\tilde{G}$-distinguished. Then for any $W \in \mathcal{W}(\pi, \psi)$

$$
\int_{\left[N^{\circ}\right]}\left|A_{1}(\pi(v) W)\right| d v<\infty
$$

and

$$
\int_{\left[N^{\mathrm{ab}}\right]}\left(\int_{\left[N^{\circ}\right]}\left|A_{1}(\pi(v u) W)\right| d v\right)^{2} d u<\infty .
$$

In the non-split case we will make the following assumption.

Assumption 2.8. Corollary 2.7 holds in the non-split case as well.

In the $p$-adic case, it is likely that this can be deduced from the results of [Lag08, KT08], but we don't address this issue here.

2.4. Observe that

$$
A_{1}(W)=\int_{\tilde{N} \backslash \tilde{\mathcal{P}}} W(g) d g
$$

since $\tilde{H}_{1} \tilde{N}=\tilde{\mathcal{P}}$. Therefore $A_{1}$ is a $\tilde{\mathcal{P}}$-invariant functional.

Suppose that $E / F$ splits and $\pi \in \Pi_{\text {gen }}(G)$ is unitarizable and $\tilde{G}_{-}$ distinguished. Then $\pi=\pi_{1} \otimes \hat{\pi}_{1}$ where $\pi_{1}$ is an irreducible unitarizable representations of $\mathrm{GL}_{n}(F)$. It is known that $A_{1}$ is $\tilde{G}$-invariant [Ber84, Bar03]. 
We can write $\psi(a, b)=\psi_{1}(a-b)$ for some character $\psi_{1}$ of $F$ and take $W$ of the form $W(x, y)=W_{1}(x) \hat{W}_{1}(y)$ with $W_{1} \in \mathcal{W}\left(\pi_{1}, \psi_{1}\right)$ and $\hat{W}_{1} \in \mathcal{W}\left(\hat{\pi}, \psi_{1}^{-1}\right)$. Then $W^{\sharp}(x, y)$ has the form $W_{2}(y) \hat{W}_{2}(x)$ with $W_{2} \in$ $\mathcal{W}\left(\pi_{1}, \psi_{1}\right)$ and $\hat{W}_{2} \in \mathcal{W}\left(\hat{\pi}, \psi_{1}^{-1}\right)$. By uniqueness we have $A_{1}\left(W^{\sharp}\right)=$ $c A_{1}(W)$ for some positive constant $c$. Since $A_{1}(W)=A_{1}\left(\left(W^{\sharp}\right)^{\sharp}\right)=$ $c A_{1}\left(W^{\sharp}\right)=c^{2} A_{1}(W)$ we get that $c^{2}=1$ and hence $c=1$. Thus,

$$
A_{1}\left(W^{\sharp}\right)=A_{1}(W) .
$$

In the non-archimedean inert case, again when $\pi$ is unitarizable and $\tilde{G}$-distinguished, it is still true by [Off11, Theorem 3.1] that $A_{1}$ is $\tilde{G}$-invariant and that (14) holds [Off11, Corollary 7.2].

We also record the following result of Matringe.

TheOREM 2.9 ([Mat11]). Suppose that $E / F$ is a quadratic extension of p-adic fields. Let $\tau$ be the Galois involution. Then the $\tilde{G}$ distinguished representations $\pi \in \Pi_{\text {gen }}(G)$ are precisely the irreducible representations of the form

$$
\pi=\pi_{1} \times \cdots \times \pi_{k} \times \hat{\sigma} \times \sigma^{\tau}
$$

where $\pi_{i} \in \Pi\left(\mathrm{GL}_{n_{i}}(E)\right), i=1, \ldots, k$ is square-integrable and $\mathrm{GL}_{n_{i}}(F)$ distinguished and $\sigma \in \Pi_{\text {gen }}\left(\mathrm{GL}_{m}(E)\right)$, where $n=n_{1}+\cdots+n_{k}+2 m$.

Assumption 2.10. Both Theorem 2.9 and the relation (14) hold for $E / F=\mathbb{C} / \mathbb{R}$.

Note that the characterization of generic irreducible representations of $\mathrm{GL}_{n}(\mathbb{C})$ which are distinguished by a unitary group was given in [FLO12]. It is likely that the methods of [loc. cit.] apply for the analogue of Theorem 2.9 in the archimedean case. So Assumption 2.10 is certainly within reach.

\section{Model transition - first case}

Throughout this section let $\pi \in \Pi_{\text {temp }}(G)$ be $\tilde{G}$-distinguished. In the non-split case we will work under Assumption 2.8. Our goal is to express the $A_{r}$ 's in terms of $A_{1}$.

\section{1.}

Lemma 3.1. Let $W \in \mathcal{W}(\pi, \psi)$ and $i=1, \ldots, n-1$. Then

(1) The function $u \mapsto A_{1}(\pi(u) W)$ lies in $L^{1}\left(\left[U_{i}^{\circ}\right]\right)$.

(2) Define the function $\mathcal{F}_{W, i}$ on $U_{i}^{\text {ab }}$ by

$$
\mathcal{F}_{W, i}(u)=\int_{\left[U_{i}^{\circ}\right]} A_{1}(\pi(v u) W) d v .
$$


Then $\mathcal{F}_{W, i} \in L^{2}\left(\left[U_{i}^{\mathrm{ab}}\right]\right)$.

Proof. It is clear that $\mathcal{F}_{W, i}$, once defined, is left invariant under $U_{i}^{\circ}$ and thus defines a function on $U_{i}^{\mathrm{ab}}$, and in fact on $\left[U_{i}^{\mathrm{ab}}\right]$ since $A_{1}$ is $\tilde{\mathcal{P}}$-invariant. We will show that for $i<n$ we have

$$
\int_{\left[U_{i}^{\circ}\right]}\left|A_{1}(\pi(v) W)\right| d v<\infty
$$

and

$$
\int_{\left[U_{i}^{\mathrm{ab}}\right]}\left(\int_{\left[U_{i}^{\circ}\right]}\left|A_{1}(\pi(v u) W)\right| d v\right)^{2} d u<\infty .
$$

In the case $i=n-1\left(U_{n-1}=N\right)$ this is precisely Corollary 2.7 and Assumption 2.8.

The $p$-adic case follows trivially from this. In the archimedean case we will prove (16) and (17) by descending induction on $i$.

Assume that (16) and (17) hold for $i=k$ and consider the case $i=k-1$. Recall that $U_{k}^{\circ}=C_{k}^{\circ} \ltimes U_{k-1}^{\circ}$. By Dixmier-Malliavin, we can assume that $W=\pi\left(f_{1}\right) W^{\prime}$ where $f_{1} \in C_{c}^{\infty}\left(C_{k}^{\circ}\right)$. Set $\tilde{f}_{1}(u)=$ $\int_{\tilde{C}_{k}^{\circ}}\left|f_{1}(v u)\right| d v \in C_{c}\left(\left[C_{k}^{\circ}\right]\right)$. Then for $u \in U_{k-1}^{\mathrm{ab}}$ :

$$
\begin{aligned}
& \int_{\left[U_{k-1}^{\circ}\right]}\left|A_{1}(\pi(v u) W)\right| d v=\int_{\left[U_{k-1}^{\circ}\right]}\left|\int_{C_{k}^{\circ}} f_{1}\left(v^{\prime}\right) A_{1}\left(\pi\left(v u v^{\prime}\right) W^{\prime}\right) d v^{\prime}\right| d v \\
\leq & \int_{C_{k}^{\circ}} \int_{\left[U_{k-1}^{\circ}\right]}\left|f_{1}\left(v^{\prime}\right) A_{1}\left(\pi\left(v u v^{\prime}\right) W^{\prime}\right)\right| d v d v^{\prime} .
\end{aligned}
$$

Since $v^{\prime}$ normalizes $U_{k-1}^{\circ}$ and commutes with $U_{k-1}$ modulo $U_{k-1}^{\circ}$ we can write this as

$$
\begin{aligned}
& \int_{C_{k}^{\circ}} \int_{\left[U_{k-1}^{\circ}\right]}\left|f_{1}\left(v^{\prime}\right) A_{1}\left(\pi\left(v v^{\prime} u\right) W^{\prime}\right)\right| d v d v^{\prime}= \\
= & \int_{\left[C_{k}^{\circ}\right]} \int_{\tilde{C}_{k}^{\circ}} \int_{\left[U_{k-1}^{\circ}\right]}\left|f_{1}\left(v_{1}^{\prime} v_{2}^{\prime}\right) A_{1}\left(\pi\left(v v_{1}^{\prime} v_{2}^{\prime} u\right) W^{\prime}\right)\right| d v d v_{1}^{\prime} d v_{2}^{\prime} .
\end{aligned}
$$

Since $v_{1}^{\prime}$ normalizes $\tilde{U}_{k-1}^{\circ}$ we get

$$
\begin{aligned}
& \int_{\left[C_{k}^{\circ}\right]} \int_{\left[U_{k-1}^{\circ}\right]} \tilde{f}_{1}\left(v^{\prime}\right)\left|A_{1}\left(\pi\left(v v^{\prime} u\right) W^{\prime}\right)\right| d v d v^{\prime} \\
\leq & \sup \tilde{f}_{1} \cdot \int_{\left[C_{k}^{\circ}\right]} \int_{\left[U_{k-1}^{\circ}\right]}\left|A_{1}\left(\pi\left(v v^{\prime} u\right) W^{\prime}\right)\right| d v d v^{\prime} \\
= & \sup \tilde{f}_{1} \cdot \int_{\left[U_{k}^{\circ}\right]}\left|A_{1}\left(\pi(v u) W^{\prime}\right)\right| d v .
\end{aligned}
$$


This proves (16) for $i=k-1$. Moreover to prove (17) for $i=k-1$, we only need to show that

$$
\int_{\left[U_{k-1}^{\mathrm{ab}}\right]}\left(\int_{\left[U_{k}^{\circ}\right]}\left|A_{1}(\pi(v u) W)\right| d v\right)^{2} d u<\infty .
$$

Assume that $W=\pi\left(f_{2}\right) W^{\prime \prime}$ where $f_{2} \in C_{c}^{\infty}\left(N_{\alpha_{n-k}}\right)$. Let

$$
\tilde{f}_{2}(u)=\int_{\tilde{N}_{\alpha_{n-k}}}\left|f_{2}(v u)\right| d v \in C_{c}\left(\left[N_{\alpha_{n-k}}\right]\right) .
$$

Then since $N_{\alpha_{n-k}}$ normalizes $U_{k-1}$ and $U_{k-1}^{\circ}$ while $\tilde{N}_{\alpha_{n-k}}$ normalizes $U_{k}^{\circ}$ and $\tilde{U}_{k}^{\circ}$ we have

$$
\begin{aligned}
& \int_{\left[U_{k-1}^{\mathrm{ab}}\right]}\left(\int_{\left[U_{k}^{\circ}\right]}\left|A_{1}(\pi(v u) W)\right| d v\right)^{2} d u \\
& \leq \int_{\left[U_{k-1}^{\mathrm{ab}}\right]}\left(\int_{\left[U_{k}^{\circ}\right]} \int_{N_{\alpha_{n-k}}}\left|A_{1}\left(\pi\left(v u u^{\prime}\right) W^{\prime \prime}\right) f_{2}\left(u^{\prime}\right)\right| d u^{\prime} d v\right)^{2} d u \\
& =\int_{\left[U_{k-1}^{\mathrm{ab}}\right]}\left(\int_{N_{\alpha_{n-k}}} \int_{\left[U_{k}^{\circ}\right]}\left|A_{1}\left(\pi\left(v u^{\prime} u\right) W^{\prime \prime}\right)\right| d v\left|f_{2}\left(u^{\prime}\right)\right| d u^{\prime}\right)^{2} d u \\
& =\int_{\left[U_{k-1}^{\mathrm{ab}}\right]}\left(\int_{\left[N_{\alpha_{n-k}}\right]} \int_{\left[U_{k}^{\circ}\right]}\left|A_{1}\left(\pi\left(v u^{\prime} u\right) W^{\prime \prime}\right)\right| d v \tilde{f}_{2}\left(u^{\prime}\right) d u^{\prime}\right)^{2} d u \\
& \leq \int_{\left[N_{\left.\alpha_{n-k}\right]}\right.} \tilde{f}_{2}\left(u^{\prime}\right)^{2} d u^{\prime} \cdot \int_{\left[U_{k-1}^{\mathrm{ab}}\right]} \int_{\left[N_{\alpha_{n-k}}\right]}\left(\int_{\left[U_{k}^{\circ}\right]}\left|A_{1}\left(\pi\left(v u^{\prime} u\right) W^{\prime \prime}\right)\right| d v\right)^{2} d u^{\prime} d u \\
& =\int_{\left[N_{\alpha_{n-k}}\right]} \tilde{f}_{2}\left(u^{\prime}\right)^{2} d u^{\prime} \cdot \int_{\left[U_{k}^{\mathrm{ab} b}\right]}\left(\int_{\left[U_{k}^{\circ}\right]}\left|A_{1}\left(\pi(v u) W^{\prime \prime}\right)\right| d v\right)^{2} d u<\infty
\end{aligned}
$$

where we used the induction hypothesis, the Cauchy-Schwarz inequality and the fact that $\left[U_{k}^{\mathrm{ab}}\right]=\left[U_{k-1}^{\mathrm{ab}}\right] \times\left[N_{\alpha_{n-k}}\right]$. This completes the induction step.

3.2. Consider now the Fourier transform of $\mathcal{F}_{W, i}$ as an $L^{2}$-function of $\widehat{\left[U_{i}^{\mathrm{ab}}\right]}$, the group of characters of $\left[U_{i}^{\mathrm{ab}}\right]$.

We denote by $\widehat{\left[U_{i}^{\mathrm{ab}}\right]_{\text {reg }}}$ the set of non-degenerate characters on $\left[U_{i}^{\mathrm{ab}}\right]$, which is an open subset of $\widehat{\left[U_{i}^{\mathrm{ab}}\right]}$. The group $\tilde{T}_{i}$ acts on $\left[U_{i}^{\mathrm{ab}}\right]$ by conjugation. Therefore it acts on $\widehat{\left[U_{i}^{\mathrm{ab}}\right]}$ and it acts simply transitively on $\widehat{\left[U_{i}^{\mathrm{ab}}\right]_{\mathrm{reg}}}$. Let

$$
\kappa=\kappa^{i}: \tilde{T}_{i} \rightarrow \widehat{\left[U_{i}^{\mathrm{ab}}\right]}
$$

be the open embedding

$$
\kappa(t)=\psi\left(t \cdot t^{-1}\right)^{-1}
$$


The image of $\kappa$ is $\widehat{\left[U_{i}^{\mathrm{ab}}\right]_{\text {reg }}}$.

For any function $f$ on $\widehat{\left[U_{i}^{\text {ab }}\right]}$ we write $\kappa^{*} f=f \circ \kappa$ (a function on $\tilde{T}_{i}$ ) and for any function $g$ on $\tilde{T}_{i}$ we write $\kappa_{*} g$ to be the extension of $g$ by 0 to $\widehat{\left[U_{i}^{\mathrm{ab}}\right]}$. Thus,

$$
\kappa_{*}: C_{c}^{\infty}\left(\tilde{T}_{i}\right) \rightarrow C_{c}^{\infty}\left(\widehat{\left[U_{i}^{\mathrm{ab}}\right]}\right) .
$$

Let $\phi \in \mathcal{S}\left(\left[U_{i}^{\mathrm{ab}}\right]\right)$, considered also as a function on $\left[U_{i}\right]$. Define

$$
\mathcal{J}_{i}(W, \phi)=\int_{\left[U_{i}^{\mathrm{ab}}\right]} \phi(u) \mathcal{F}_{W, i}(u) d u=\int_{\left[U_{i}\right]} \phi(u) A_{1}(\pi(u) W) d u .
$$

Set $S_{i}=\tilde{T}_{i} \ltimes\left[U_{i}^{\circ}\right]$. Note that for $t \in \tilde{T}_{i}$,

$$
\delta_{S_{i}}(t)=\left|t_{n-i}\right|^{i-1} \prod_{j=n-i+1}^{n-1}\left|t_{j}\right|^{n-2 j+1} .
$$

LEMma 3.2. Let $f \in C_{c}^{\infty}\left(\tilde{T}_{i}\right)$ and $W^{\prime}=\pi\left(f \delta_{S_{i}}^{-1}\right) W$. Then $\kappa^{*} \widehat{\mathcal{F}_{W^{\prime}, i}}$ is given by $t \mapsto\left|t_{n-i}^{-1}\right| \mathcal{J}_{i}\left(\pi(t) W, \widehat{\kappa_{*} f}\right)$. In particular, it is a smooth function on $\tilde{T}_{i}$.

Proof. We have

$$
\begin{aligned}
& \mathcal{F}_{W^{\prime}, i}(u)=\int_{\left[U_{i}^{\circ}\right]} \int_{\tilde{T}_{i}} f\left(t^{\prime}\right) \delta_{S_{i}}^{-1}\left(t^{\prime}\right) A_{1}\left(\pi\left(v u t^{\prime}\right) W\right) d t^{\prime} d v \\
& =\int_{\left[U_{i}^{\circ}\right]} \int_{\tilde{T}_{i}} f\left(t^{\prime}\right) A_{1}\left(\pi\left(v t^{\prime-1} u t^{\prime}\right) W\right) d t^{\prime} d v=\int_{\tilde{T}_{i}} f\left(t^{\prime}\right) \mathcal{F}_{W, i}\left(t^{\prime-1} u t^{\prime}\right) d t^{\prime} .
\end{aligned}
$$

It is easy to see that the value of the Fourier transform of $\mathcal{F}_{W, i}\left(t^{\prime-1} \cdot t^{\prime}\right)$ at $\kappa(t)$ is $\left|\frac{t_{n-i}^{\prime}}{t_{n-i}}\right| \kappa^{*} \widehat{\mathcal{F}_{\pi(t) W, i}}\left(t^{\prime}\right)$. Note that the push-forward of the measure $d^{a} t^{\prime}=\left|t_{n-i}^{\prime}\right| d t^{\prime}$ on $\tilde{T}_{i}$ via $\kappa$ is the dual measure to $d u$ on $\left[U_{i}^{\mathrm{ab}}\right]$. Thus, by Parseval's identity, the value of the Fourier transform of $\mathcal{F}_{W^{\prime}, i}$ at $\kappa(t)$ is

$$
\begin{aligned}
& \left|t_{n-i}^{-1}\right| \int_{\tilde{T}_{i}} f\left(t^{\prime}\right) \kappa^{*} \widehat{\mathcal{F}_{\pi(t) W, i}}\left(t^{\prime}\right) d^{a} t^{\prime}= \\
& \left|t_{n-i}^{-1}\right| \int_{\left[U_{i}^{\mathrm{ab}}\right]} \widehat{\kappa_{*} f}(u) \mathcal{F}_{\pi(t) W, i}(u) d u=\left|t_{n-i}^{-1}\right| \mathcal{J}_{i}\left(\pi(t) W, \widehat{\kappa_{*} f}\right)
\end{aligned}
$$

as claimed.

Define $\mathcal{E}_{i}(W)$ to be the value of the Fourier transform of $\mathcal{F}_{W, i}$ at $\psi^{-1}$. Thus $\mathcal{E}_{i}(W)$ is a regularization of the integral

$$
\mathcal{E}_{i}(W)=\int_{\left[U_{i}\right]} A_{1}(\pi(u) W) \psi(u)^{-1} d u
$$


which is in general not absolutely convergent. Note that $\mathcal{E}_{0}(W)=$ $A_{1}(W)$.

REMARK 3.3. We expect that in the non-archimedean case, the integral

$$
\int_{\left[U_{i}\right]} A_{1}(\pi(u) W) \psi(u)^{-1} d u
$$

makes sense as a stable integral, i.e. stabilizes for large enough compact open subgroups of $\left[U_{i}\right]$. However, we will not address this issue here.

REMARK 3.4. One can define $\mathcal{E}_{i}(W)$ (in the tempered case) without knowing that $\mathcal{F}_{W, i} \in L^{2}\left(\left[U_{i}^{\mathrm{ab}}\right]\right)$. Namely, one can show that the restriction of the Fourier transform of $\mathcal{F}_{W, i}$ (as a distribution) to $\widehat{\left[U_{i}^{\mathrm{ab}}\right]_{\text {reg }}}$ is smooth. Therefore, in the non-split case we would only need to know the first part of Corollary 2.7.

By Lemma 3.2 we have

$$
\mathcal{E}_{i}\left(\pi\left(f \delta_{S_{i}}^{-1}\right) W\right)=\mathcal{J}_{i}\left(W, \widehat{\kappa_{*} f}\right) .
$$

The rest of this section is devoted to the proof of the following result.

Proposition 3.5. For $i=1, \ldots, n$ we have

$$
A_{i}(W)=\mathcal{E}_{i-1}(W)
$$

for any $W \in \mathcal{W}(\pi, \psi)$.

Fix $i=0, \ldots, n-1$. By (20) it suffices to prove that for all $f \in$ $C_{c}^{\infty}\left(\tilde{T}_{i}\right)$ we have

$$
A_{i+1}\left(\pi\left(f \delta_{S_{i}}^{-1}\right) W\right)=\mathcal{J}_{i}\left(W \widehat{\kappa_{*} f}\right)
$$

REMARK 3.6. Since $A_{i+1}(\pi(\cdot) W)$ is a distribution on $C_{c}^{\infty}\left(\tilde{T}_{i}\right)$ and $\mathcal{J}_{i}(W, \cdot)$ is a tempered distribution on $\mathcal{S}\left(\left[U_{i}^{\mathrm{ab}}\right]\right)$ it suffices to prove (21) for functions of the form $f(t)=\prod_{k=1}^{i} \phi_{k}\left(\frac{t_{n-k}}{t_{n-k+1}}\right)$ when $t \in \tilde{T}_{i}$, with $\phi_{k} \in C_{c}^{\infty}\left(F^{*}\right)$.

3.3. To prove (21), we use induction on $i$. The case $i=0$ is trivial $\left(T_{0}=1\right)$.

By Remark 3.6, we can assume $f(t)=f_{1}(t) f_{2}(t)$, where $f_{2} \in$ $C_{c}^{\infty}\left(\tilde{T}_{i-1}\right)$ considered as a function on $\tilde{T}_{i}$ via the obvious projection $\tilde{T}_{i} \rightarrow \tilde{T}_{i-1}$ and $f_{1}(t)=\phi\left(\alpha_{n-i}(t)\right)=\phi\left(\frac{t_{n-i}}{t_{n-i+1}}\right)$ with $\phi \in C_{c}^{\infty}\left(F^{*}\right)$.

Let $\jmath: \tilde{T}_{i} \rightarrow\left[\widehat{N_{\alpha_{n-i}}}\right]$ be the map $t \mapsto \psi\left(t \cdot t^{-1}\right)^{-1}$, which factors through $\alpha_{n-i}$. For any function $g$ on $\tilde{T}_{i}$ which depends only on $\alpha_{n-i}(t)$ 
let $\jmath_{*} g$ be the function on $\left[\widehat{N_{\alpha_{n-i}}}\right]$ which is 0 on the trivial character and given by $g(t)$ on $\jmath(t)$.

Then $\widehat{\kappa_{*}^{i f f}}=\widehat{\jmath_{*} f_{1}} \widehat{\kappa_{*}^{i-1} f_{2}}$ where we consider $\widehat{\kappa_{*}^{i-1} f_{2}}$ as function on $\left[U_{i}^{\mathrm{ab}}\right]$ that factors through $\left[U_{i-1}^{\mathrm{ab}}\right]$, and $\widehat{J_{*} f_{1}}$ factors through $\left[N_{\alpha_{n-i}}\right]$. More precisely, $\widehat{\jmath_{*} f_{1}}\left(\lambda_{n-i}(x)\right)=\hat{\phi}(x)$ for $x \in F \backslash E$. We will consider $\widehat{\jmath_{*} f_{1}}$ as a function on $\left[C_{i}\right]$ as $C_{i}=N_{\alpha_{n-i}} \times C_{i}^{\circ}$.

Recall that $U_{i}=C_{i} \ltimes U_{i-1}$. By induction hypothesis we have

$$
\begin{aligned}
\mathcal{J}_{i}\left(W, \widehat{\kappa^{*} f}\right) & =\int_{\left[C_{i}\right]} \widehat{\jmath_{*} f_{1}}(u) \mathcal{J}_{i-1}\left(\pi(u) W, \widehat{\kappa_{*}^{i-1} f_{2}}\right) d u \\
& =\int_{\left[C_{i}\right]} \widehat{J_{*} f_{1}}(u) A_{i}\left(\pi\left(f_{2} \delta_{S_{i-1}}^{-1}\right) \pi(u) W\right) d u \\
& =\int_{\left[C_{i}\right]} \widehat{\jmath_{*} f_{1}}(u)\left(\int_{\tilde{T}_{i-1}} f_{2}(t) \delta_{S_{i-1}}(t)^{-1} A_{i}(\pi(t u) W) d t\right) d u .
\end{aligned}
$$

We will express $A_{i}$ in terms of $A_{i+1}$. Let $T_{i}^{0} \subset T_{i}$ be the onedimensional subtorus consisting of $\operatorname{diag}\left(t_{1}, \ldots, t_{n}\right)$ with $t_{k}=1$ if $k \neq$ $n-i$. Observe that the map

$$
\left(\tilde{N}_{H_{i+1}} \backslash \tilde{H}_{i+1} \times \tilde{T}_{i}^{0}\right) \ltimes \tilde{D}_{i} \rightarrow \tilde{N}_{H_{i}} \backslash \tilde{H}_{i}
$$

is an open embedding with dense image. Thus,

$$
A_{i}(W)=\int_{\tilde{T}_{i}^{0}} \int_{\tilde{D}_{i}} A_{i+1}(\pi(\xi t) W)|\operatorname{det} t|^{1-i} d \xi d t .
$$

Therefore, we can write $\mathcal{J}_{i}\left(W, \widehat{\kappa_{*} f}\right)$ as

$$
\int_{\left[C_{i}\right]}\left(\int_{\tilde{T}_{i-1}} \int_{\tilde{T}_{i}^{0}} \int_{\tilde{D}_{i}} \widehat{J_{*} f_{1}}(u) f_{2}(t) \delta_{S_{i-1}}^{-1}(t) A_{i+1}\left(\pi\left(\xi t^{\prime} t u\right) W\right)\left|t_{n-i}^{\prime}\right|^{1-i} d \xi d t^{\prime} d t\right) d u .
$$

From (19) we get for $t^{\prime \prime}=t t^{\prime}$ with $t \in \tilde{T}_{i-1}$ and $t^{\prime} \in \tilde{T}_{i}^{0}$ :

$$
\delta_{S_{i-1}}(t)^{-1}\left|t_{n-i}^{\prime}\right|^{1-i}\left|t_{n-i+1}\right|^{n-i-1}=\delta_{S_{i}}\left(t^{\prime \prime}\right)^{-1} .
$$

As $\tilde{T}_{i}=\tilde{T}_{i-1} \times \tilde{T}_{i}^{0}, \mathcal{J}_{i}\left(W, \widehat{\kappa_{*} f}\right)$ equals

$$
\int_{\left[C_{i}\right]}\left(\int_{\tilde{T}_{i}} \int_{\tilde{D}_{i}} \widehat{J_{*} f_{1}}(u) f_{2}(t) \delta_{S_{i}}^{-1}(t) A_{i+1}(\pi(\xi t u) W)\left|t_{n-i+1}\right|^{1+i-n} d \xi d t\right) d u .
$$

Write $u \in C_{i}$ as $u_{1} u_{2}$ with $u_{1} \in C_{i}^{\circ}$ and $u_{2} \in N_{\alpha_{n-i}}$. Then the above integration can be written as

$$
\int_{\left[C_{i}^{\circ}\right]} \int_{\left[N_{\left.\alpha_{n-1}\right]}\right]}\left(\int_{\tilde{T}_{i}} \int_{\tilde{D}_{i}}\right)=\int_{\left[C_{i}^{\circ}\right]}\left(\int_{\left[N_{\alpha_{n-1}}\right]} \int_{\tilde{T}_{i}} \int_{\tilde{D}_{i}}\right)
$$


as the inner triple integral is absolutely convergent. It is clear from (5) that:

$$
A_{i+1}(\pi(u) W)=\psi(u) A_{i+1}(W), \quad u \in U_{i} .
$$

Thus $A_{i+1}\left(\pi\left(\xi t u_{1} u_{2}\right) W\right)=A_{i+1}(\pi(\xi t) W)\left\langle t u_{1} t^{-1}, \xi\right\rangle \psi\left(t u_{2} t^{-1}\right)$. We get

$$
\begin{aligned}
& \mathcal{J}_{i}\left(W, \widehat{\kappa_{*} f}\right)=\int_{\left[C_{i}^{\circ}\right]}\left(\int_{\left[N_{\left.\alpha_{n-1}\right]}\right]} \int_{\tilde{T}_{i}} \int_{\tilde{D}_{i}} \widehat{J_{*} f_{1}}\left(u_{2}\right) f_{2}(t) \delta_{S_{i}}^{-1}(t)\right. \\
& \left.A_{i+1}(\pi(\xi t) W)\left|t_{n-i+1}\right|^{1+i-n}\left\langle t u_{1} t^{-1}, \xi\right\rangle \psi\left(t u_{2} t^{-1}\right) d \xi d t d u_{2}\right) d u_{1} .
\end{aligned}
$$

Integrating first over $u_{2}$ and using Fourier inversion, $\mathcal{J}_{i}\left(W \widehat{\kappa_{*} f}\right)$ equals $\int_{\left[C_{i}^{\circ}\right]}\left(\int_{\tilde{T}_{i}} \int_{\tilde{D}_{i}} f(t) \delta_{S_{i}}^{-1}(t) A_{i+1}(\pi(\xi t) W)\left|t_{n-i+1}\right|^{1+i-n}\left\langle t u_{1} t^{-1}, \xi\right\rangle d \xi d t\right) d u_{1}$.

It will follow from Lemma 3.8 below that we can switch the order of integration, and integrate over $\left[C_{i}^{\circ}\right]$ before the integration over $\tilde{T}_{i}$. Changing $u_{1}$ to $t^{-1} u_{1} t$ we get:

$\mathcal{J}_{i}\left(W, \widehat{\kappa_{*} f}\right)=\int_{\tilde{T}_{i}} \int_{\left[C_{i}^{\circ}\right]}\left(\int_{\tilde{D}_{i}} f(t) \delta_{S_{i}}^{-1}(t) A_{i+1}(\pi(\xi t) W)\left\langle u_{1}, \xi\right\rangle d \xi\right) d u_{1} d t$.

By Lemma 3.7 below $A_{i+1}(\pi(\xi) W) \in \mathcal{S}\left(\tilde{D}_{i}\right)$. Hence by Fourier inversion the above is:

$$
\left.\mathcal{J}_{i}\left(W, \widehat{\kappa_{*} f}\right)=\int_{\tilde{T}_{i}} f(t) \delta_{S_{i}}^{-1}(t) A_{i+1}(\pi(t) W) d t=A_{i+1}\left(\pi\left(f \delta_{S_{i}}^{-1}\right)\right) W\right) .
$$

This completes the induction step and thus the proof of Proposition 3.5.

It remains to show the following two Lemmas.

Lemma 3.7. The function $\xi \mapsto A_{i+1}(\pi(\xi) W)$ on $D_{i}$ lies in $\mathcal{S}\left(D_{i}\right)$.

LEMma 3.8. For any $f \in C_{c}^{\infty}\left(\tilde{T}_{i}\right)$,

$$
\int_{\left[C_{i}^{\circ}\right]} \int_{\tilde{T}_{i}}\left(\int_{\tilde{D}_{i}} f(t) A_{i+1}(\pi(\xi t) W)\left\langle t u_{1} t^{-1}, \xi\right\rangle d \xi\right) d t d u_{1}
$$

is absolutely convergent.

Proof of Lemma 3.7. A priori $A_{i+1}(\pi(\cdot) W)$ is a tempered function on $D_{i}$. We can assume $W=\pi\left(\phi^{\prime}\right) W^{\prime}$ where $\phi^{\prime} \in C_{c}^{\infty}\left(C_{i}^{\circ}\right)$. It follows from (5) that

$$
\begin{aligned}
A_{i+1}(\pi(\xi) W) & =\int_{C_{i}^{\circ}} A_{i+1}\left(\pi(\xi u) W^{\prime}\right) \phi^{\prime}(u) d u \\
& =\int_{C_{i}^{\circ}} A_{i+1}\left(\pi(\xi) W^{\prime}\right) \phi^{\prime}(u)\langle u, \xi\rangle d u=A_{i+1}\left(\pi(\xi) W^{\prime}\right) \hat{\phi}^{\prime}(\xi) .
\end{aligned}
$$

The Lemma follows. 
Proof of Lemma 3.8. We can assume $W=\pi\left(f^{\prime}\right) W^{\prime}$ where $f^{\prime} \in$ $C_{c}^{\infty}\left(\tilde{D}_{i}\right)$. Then we need to consider

$$
\int_{\left[C_{i}^{\circ}\right]} \int_{\tilde{T}_{i}}\left|\left(\int_{\tilde{D}_{i}} \int_{\tilde{D}_{i}} f(t) f^{\prime}\left(\xi^{\prime}\right) A_{i+1}\left(\pi\left(\xi t \xi^{\prime}\right) W^{\prime}\right)\left\langle t u_{1} t^{-1}, \xi\right\rangle d \xi^{\prime} d \xi\right)\right| d t d u_{1} .
$$

By changing $\xi \mapsto \xi t\left(\xi^{\prime}\right)^{-1} t^{-1}$ and using (23), the inner double integral inside the absolute value is

$$
\int_{\tilde{D}_{i}} \int_{\tilde{D}_{i}} f(t) f^{\prime}\left(\xi^{\prime}\right) A_{i+1}\left(\pi\left(\xi t u_{1}\right) W^{\prime}\right)\left\langle t u_{1} t^{-1}, t\left(\xi^{\prime}\right)^{-1} t^{-1}\right\rangle d \xi^{\prime} d \xi
$$

Integrating over $\xi^{\prime}$ we can express the above as

$$
\int_{\left[C_{i}^{\circ}\right] \rtimes \tilde{T}_{i}}\left|\left(\int_{\tilde{D}_{i}} f^{\prime \prime}(g) A_{i+1}\left(\pi(\xi g) W^{\prime}\right) d \xi\right)\right| d g
$$

for some Schwartz function $f^{\prime \prime}$ on $\left[C_{i}^{\circ}\right] \rtimes \tilde{T}_{i}$. The Lemma now follows from the previous one.

\section{Model transition - second case}

4.1. Throughout this section let $\pi \in \Pi_{\text {temp }}(G)$ be $\tilde{G}$-distinguished. Our goal is to express $A_{r}^{\prime}(W)$ (defined in $(2)$ ) in terms of $A_{1}^{\prime}(W)=$ $A_{1}(W)$. We keep the proviso of Assumptions 2.8 and 2.10.

Let $N_{G_{r}}=N \cap G_{r}$. Define the function $\mathcal{F}_{W, r}^{\prime}$ on $\left[N_{G_{r}}^{\mathrm{ab}}\right]$ by

$$
\mathcal{F}_{W, r}^{\prime}(u)=\int_{\left[R_{r} \rtimes N_{G_{r}}^{\circ}\right]} A_{1}(\pi(v u) W) d v .
$$

LEMma 4.1. Let $\hat{w}_{r}:=\left(\begin{array}{ll}I_{n-r} & \\ { }_{r}\end{array}\right)=w_{n, r}^{*} w_{n}$, and let $u \mapsto \hat{u}$ be the automorphism of $N_{G_{r}}$ given by $\hat{u}=\hat{w}_{r} u^{\sigma} \hat{w}_{r}^{-1}$. Then for $W \in \mathcal{W}(\pi, \psi)$ we have the identity $\mathcal{F}_{W^{\sharp}, r-1}(u)=\mathcal{F}_{\pi\left(\hat{w}_{r}\right) W, r}^{\prime}(\hat{u}), u \in N_{G_{r}}$. In particular,

(1) The integral defining $\mathcal{F}_{W, r}^{\prime}$ is absolutely convergent.

(2) $\mathcal{F}_{W, r}^{\prime} \in L^{2}\left(\left[N_{G_{r}}^{\mathrm{ab}}\right]\right)$.

(3) The Fourier transform of $\mathcal{F}_{W, r}^{\prime}$ is smooth at the non-degenerate elements of $\widehat{\left[N_{G_{r}}^{\mathrm{ab}}\right]}$. Let $\mathcal{E}_{r}^{\prime}(W)=\widehat{\mathcal{F}_{W, r}^{\prime}}\left(\psi^{-1}\right)$. Then $\mathcal{E}_{r}^{\prime}\left(\pi\left(\hat{w}_{r}\right) W\right)=$ $\mathcal{E}_{r-1}\left(W^{\sharp}\right)$.

Proof. Recall that $W^{\sharp} \in \mathcal{W}\left(\hat{\pi}, \psi^{-1}\right)$. Thus by (15)

$$
\begin{aligned}
\mathcal{F}_{W^{\sharp}, r-1}(u) & =\int_{\left[U_{r-1}^{\circ}\right]} A_{1}\left(\hat{\pi}(v u) W^{\sharp}\right) d v \\
= & \int_{\left[U_{r-1}^{\circ}\right]} A_{1}\left(\left[\pi\left(v^{\sigma} u^{\sigma}\right) W\right]^{\sharp}\right) d v=\int_{\left[U_{r-1}^{\circ}\right]} A_{1}\left(\pi\left(v^{\sigma} u^{\sigma}\right) W\right) d v,
\end{aligned}
$$


where the last equation follows from the relation (14). Since $\hat{w}_{r}\left(U_{r-1}^{\circ}\right)^{\sigma} \hat{w}_{r}^{-1}=$ $R_{r} \rtimes N_{G_{r}}^{\circ}$, we deduce that the above is

$$
\int_{\left[R_{r} \rtimes N_{G_{r}}^{\circ}\right]} A_{1}\left(\pi\left(\hat{w}_{r} v \hat{u} \hat{w}_{r}\right) W\right) d v=\mathcal{F}_{\pi\left(\hat{w}_{r}\right) W, r}^{\prime}(\hat{u}) .
$$

The two claims on convergence follow. The last claim $\mathcal{E}_{r}^{\prime}\left(\pi\left(\hat{w}_{r}\right) W\right)=$ $\mathcal{E}_{r-1}\left(W^{\sharp}\right)$ follows from the fact that $\psi^{-1}(\hat{u})=\psi(u)$. (Note that $\mathcal{E}_{r}\left(W^{\sharp}\right)$ is the Fourier transform of $\mathcal{F}_{W^{\sharp}, r}$ at $\psi$ because $W^{\sharp} \in \mathcal{W}\left(\hat{\pi}, \psi^{-1}\right)$.)

The goal of this section is to prove:

Proposition 4.2. For $r=1, \ldots, n-1$ we have

$$
A_{r}^{\prime}(W)=\mathcal{E}_{r}^{\prime}(W) .
$$

4.2. In order to prove the proposition we will prove a more general auxiliary statement (Proposition 4.4 below). From now on we fix a positive integer $r<n$.

Let $1 \leq i \leq r-1$. Let ${ }_{i} U_{r}=U_{i} \cap G_{r}$, then ${ }_{i} U_{r}^{\text {ab }}={ }_{i} U_{r}^{\circ} \backslash{ }_{i} U_{r}$ is an $i$-dimensional vector space. Let

$$
\mathcal{F}_{W, r, i}^{\prime}(u)=\int_{\left[R_{r} \rtimes_{i} U_{r}^{\circ}\right]} A_{1}(\pi(v u) W) d v, \quad u \in\left[{ }_{i} U_{r}^{\mathrm{ab}}\right] .
$$

We have:

Lemma 4.3. (1) The integral defining $\mathcal{F}_{W, r, i}^{\prime}$ is absolutely convergent.

(2) $\mathcal{F}_{W, r, i}^{\prime} \in L^{2}\left(\left[{ }_{i} U_{r}^{\mathrm{ab}}\right]\right)$.

(3) The Fourier transform of $\mathcal{F}_{W, r, i}^{\prime}$ is smooth at the non-degenerate elements of $\widehat{\left[{ }_{i} \widehat{\left.U_{r}^{\mathrm{ab}}\right]}\right.}$.

Proof. From the argument in Lemma 4.1 and the bounds (16) and (17), we get:

$$
\int_{\left[R_{r} \rtimes N_{G_{r}}^{\circ}\right]}\left|A_{1}(\pi(v u) W)\right| d v<\infty
$$

and

$$
\int_{\left[N_{G_{r}}^{\mathrm{ab}}\right]}\left(\int_{\left[R_{r} \rtimes N_{G_{r}}^{\circ}\right]}\left|A_{1}(\pi(v u) W)\right| d v\right)^{2} d u<\infty .
$$

Since $N_{G_{r}}={ }_{r-1} U_{r}$, we get the claim of convergence for $i=r-1$. The same induction argument as in the proof of Lemma 3.1 gives the claims of convergence. The proof of the last smoothness claim is also identical to the proof of Lemma 3.2. 
Define $\mathcal{E}_{r, i}^{\prime}(W)=\widehat{\mathcal{F}_{W, r, i}^{\prime}}\left(\psi^{-1}\right)$. Clearly, $\mathcal{E}_{r}^{\prime}=\mathcal{E}_{r, r-1}^{\prime}$.

Define the integral

$$
A_{r, i}^{\prime}(W)=\int_{\tilde{R}_{r, i+1} \backslash R_{r}} A_{i+1}(\pi(x) W) d x .
$$

It is clear that $A_{r}^{\prime}=A_{r, r-1}^{\prime}$ (since $R_{r, r}=1$ ). Thus, Proposition 4.2 is a special case of the following result which will be proved in the rest of the section.

Proposition 4.4. For any $i=0, \ldots, r-1$ the integrals defining $A_{r, i}^{\prime}(W)$ are absolutely convergent, and

$$
A_{r, i}^{\prime}(W)=\mathcal{E}_{r, i}^{\prime}(W) .
$$

4.3. As in $\S 3.2$, we can write $\mathcal{E}_{r, i}^{\prime}(W)$ using an absolutely convergent integral. For $\phi \in \mathcal{S}\left(\left[{ }_{i} U_{r}^{\mathrm{ab}}\right]\right)$ regarded as a function of $\left[R_{r} \rtimes_{i} U_{r}\right]$, define

$$
\mathcal{J}_{i}^{\prime}(W, \phi)=\int_{\left[{ }_{i} U_{r}^{\mathrm{ab}}\right]} \phi(u) \mathcal{F}_{W, r, i}^{\prime}(u) d u=\int_{\left[R_{r} \rtimes_{i} U_{r}\right]} \phi(u) A_{1}(\pi(u) W) d u .
$$

Then for $f \in C_{c}^{\infty}\left(\tilde{T}_{i}\right)$, by the same argument as in $\S 3.2$ we have

$$
\mathcal{E}_{r, i}^{\prime}\left(\pi\left(f \delta_{S_{i}^{\prime}}^{-1}\right) W\right)=\mathcal{J}_{i}^{\prime}\left(W, \widehat{\kappa_{*} f}\right)
$$

where $\left.S_{i}^{\prime}=\tilde{T}_{i} \ltimes{ }_{i} U_{r}^{\circ} \ltimes R_{r}\right]$. Note that the embedding ${ }_{i} U_{r} \hookrightarrow U_{i}$ induces an isomorphism ${ }_{i} U_{r}^{\mathrm{ab}} \simeq U_{i}^{\mathrm{ab}}$. We can therefore view $\kappa$ as a map $\kappa$ : $\tilde{T}_{i} \rightarrow \widehat{\left[{ }_{i} U_{r}^{\text {ab }}\right]}$. Note that for $t \in \tilde{T}_{i}$ :

$$
\delta_{S_{i}^{\prime}}(t)=|\operatorname{det} t|^{n-r}\left|t_{n-i}\right|^{i-1} \prod_{j=1}^{i-1}\left|t_{n-j}\right|^{-r+1+2 j} .
$$

Thus, it suffices to show that for any $i=0, \ldots, r-1$ and $f \in$ $C_{c}^{\infty}\left(\tilde{T}_{i}\right)$, the integrals

$$
A_{r, i}^{\prime}\left(\pi\left(f \delta_{S_{i}^{\prime}}^{-1}\right) W\right)=\int_{\tilde{R}_{r, i+1} \backslash R_{r}} \int_{\tilde{T}_{i}} A_{i+1}(\pi(x t) W) f(t) \delta_{S_{i}^{\prime}}^{-1}(t) d t d x
$$

are absolutely convergent and equal $\mathcal{J}_{i}^{\prime}\left(W, \widehat{\kappa_{*} f}\right)$.

4.4. We use induction on $i$, the case $i=0$ is a tautology. By Remark 3.6 we may assume that $f(t)=f_{1}(t) f_{2}(t)$ with $f_{2} \in C_{c}^{\infty}\left(\tilde{T}_{i-1}\right)$ and $f_{1}(t)=\phi^{\prime}\left(\alpha_{n-i}(t)\right)$ where $\alpha_{n-i}(t)=\frac{t_{n-i}}{t_{n-i+1}}$ and $\phi^{\prime} \in C_{c}^{\infty}\left(F^{*}\right)$.

Note that ${ }_{i} U_{r}={ }_{i-1} U_{r} \rtimes{ }_{i} C_{r}$, thus ${ }_{i} U_{r}^{\mathrm{ab}}={ }_{i-1} U_{r}^{\mathrm{ab}} \times{ }_{i} C_{r}^{\mathrm{ab}} ; \widehat{\kappa_{*} f}=$ $\widehat{J_{*} f_{1}} \widehat{\kappa_{*}^{i-1} f_{2}}$ where $\widehat{\kappa_{*}^{i-1} f_{2}}$ is a function on $\left[{ }_{i} U_{r}^{\mathrm{ab}}\right]$ that factors through $\left[{ }_{i-1} U_{r}^{\text {ab }}\right]$ and $\widehat{\jmath_{*} f_{1}}$ factors through $\left[N_{\alpha_{n-i}}\right]$. More precisely, $\widehat{\jmath_{*} f_{1}}\left(\lambda_{n-i}(x)\right)=$ 
$\widehat{\phi^{\prime}}(x)$ for $x \in F \backslash E$. We will consider $\widehat{\jmath_{*} f_{1}}$ as a function on $\left[{ }_{i} C_{r}\right]$ via ${ }_{i} C_{r}=N_{\alpha_{n-i}} \times{ }_{i} C_{r}^{\circ}$.

For the induction step we write

$$
\begin{aligned}
\mathcal{J}_{i}^{\prime}\left(W \widehat{\kappa_{*} f}\right) & =\int_{\left[R_{r} \rtimes_{i} U_{r}\right]} \widehat{\kappa_{*} f}(u) A_{1}(\pi(u) W) d u \\
& =\int_{\left[{ }_{i} C_{r}\right]} \int_{\left[R_{r} \rtimes_{i-1} U_{r}\right]} \widehat{J_{*} f_{1}}(u) \widehat{\kappa_{*}^{i-1} f_{2}}(v) A_{1}(\pi(v u) W) d v d u .
\end{aligned}
$$

By the induction hypothesis this is equal to

$$
\begin{array}{r}
\left.\int_{{ }_{\left.{ }_{i} C_{r}\right]} \widehat{J_{*} f_{1}}(u)\left(\int_{\tilde{R}_{r, i} \backslash R_{r}} \int_{\tilde{T}_{i-1}} A_{i}(\pi(x t u) W)\right.} f_{2}(t) \delta_{S_{i-1}^{\prime}}^{-1}(t) d t d x\right) d u \\
=\int_{\left[_{i} C_{r}^{\circ}\right]} \Lambda(\pi(v) W) d v
\end{array}
$$

where $\Lambda(W)$ is the following absolutely convergent triple integral:

$$
\int_{\left[N_{\alpha_{n-i}}\right]} \widehat{\jmath_{*} f_{1}}\left(u_{1}\right) \int_{\tilde{R}_{r, i} \backslash R_{r}} \int_{\tilde{T}_{i-1}} A_{i}\left(\pi\left(x t u_{1}\right) W\right) f_{2}(t) \delta_{S_{i-1}^{\prime}}^{-1}(t) d t d x d u_{1} .
$$

\section{5 .}

LEMMA 4.5. We have

$$
\begin{aligned}
& \Lambda(W)=\int_{\left(D_{r, i} \tilde{R}_{r, i}\right) \backslash R_{r}}\left(\int_{\tilde{T}_{i}} \int_{D_{r, i}} \int_{i \tilde{D}_{r}}\right. \\
& \left.A_{i+1}(\pi(\xi y x t) W) f(t) \delta_{S_{i}^{\prime}}^{-1}(t)\left|t_{n-i+1}\right|^{1+i-r} d \xi d y d t\right) d x .
\end{aligned}
$$

Proof. Note that $N_{\alpha_{n-i}}$ and $R_{r, i}$ commute. Making a change of variable $x \mapsto\left(t u_{1} t^{-1}\right) x\left(t u_{1}^{-1} t^{-1}\right)$ in (26) we get that $\Lambda(W)$ equals

$$
\int_{\tilde{R}_{r, i} \backslash R_{r}} \int_{\tilde{T}_{i-1}} \int_{\left[N_{\alpha_{n-i}}\right]} \widehat{J_{*} f_{1}}\left(u_{1}\right) A_{i}\left(\pi\left(t u_{1} t^{-1} x t\right) W\right) f_{2}(t) \delta_{S_{i-1}^{\prime}}^{-1}(t) d u_{1} d t d x .
$$

By (22) this is

$$
\begin{aligned}
\int_{\tilde{R}_{r, i} \backslash R_{r}} \int_{\tilde{T}_{i-1}} \int_{\left[N_{\alpha_{n-i}}\right]} \widehat{\widehat{J_{*} f_{1}}}\left(u_{1}\right) & \left(\int_{\tilde{T}_{i}^{0}} \int_{\tilde{D}_{i}} A_{i+1}\left(\pi\left(\xi t^{\prime} t u_{1} t^{-1} x t\right) W\right)\right. \\
& \left.f_{2}(t) \delta_{S_{i-1}^{\prime}}^{-1}(t)\left|t_{n-i}^{\prime}\right|^{1-i} d \xi d t^{\prime}\right) d u_{1} d t d x .
\end{aligned}
$$

Note that $\xi$ and $t^{\prime} t u_{1}\left(t^{\prime} t\right)^{-1}$ commute. Applying (23) we get

$$
A_{i+1}\left(\pi\left(\xi t^{\prime} t u_{1} t^{-1} x t\right) W\right)=A_{i+1}\left(\pi\left(\xi t^{\prime} x t\right) W\right) \psi\left(t^{\prime} t u_{1}\left(t^{\prime} t\right)^{-1}\right) .
$$


It is now clear that the quadruple integral over $t^{\prime}, t^{\prime}, \xi, u_{1}$ is absolutely convergent, i.e.

$$
\begin{aligned}
& \int_{\tilde{T}_{i-1}} \int_{\left[N_{\alpha_{n-i}}\right]} \int_{\tilde{T}_{i}^{0}} \int_{\tilde{D}_{i}} \\
& \left.\quad\left|A_{i+1}\left(\pi\left(\xi t^{\prime} x t\right) W\right) \widehat{\jmath_{*} f_{1}}\left(u_{1}\right) f_{2}(t) \delta_{S_{i-1}^{\prime}}^{-1}(t)\right| t_{n-i}^{\prime}\right|^{1-i} \mid d \xi d t^{\prime} d u_{1} d t<\infty .
\end{aligned}
$$

We can integrate over $u_{1}$ first. By Fourier inversion we get:

$$
\begin{aligned}
\Lambda(W)=\int_{\tilde{R}_{r, i} \backslash R_{r}} & \left(\int_{\tilde{T}_{i-1}} \int_{\tilde{T}_{i}^{0}} \int_{\tilde{D}_{i}}\right. \\
& \left.A_{i+1}\left(\pi\left(\xi t^{\prime} x t\right) W\right) f\left(t t^{\prime}\right) \delta_{S_{i-1}^{\prime}}^{-1}(t)\left|t_{n-i}^{\prime}\right|^{1-i} d \xi d t^{\prime} d t\right) d x .
\end{aligned}
$$

Next, we write $x \in \tilde{R}_{r, i} \backslash R_{r}$ as $x^{\prime} y^{\prime}$ with $y^{\prime} \in\left[D_{r, i}\right]$ and $x^{\prime} \in$ $\left(D_{r, i} \tilde{R}_{r, i}\right) \backslash R_{r}$. It follows from Lemma 3.7 that

$$
\begin{aligned}
\Lambda(W)=\int_{\left(D_{r, i} \tilde{R}_{r, i}\right) \backslash R_{r}}\left(\int_{\left[D_{r, i}\right]} \int_{\tilde{T}_{i-1}} \int_{\tilde{T}_{i}^{0}} \int_{\tilde{D}_{i}} A_{i+1}\left(\pi\left(\xi t^{\prime} y^{\prime} x^{\prime} t\right) W\right)\right. \\
\left.\quad f\left(t t^{\prime}\right) \delta_{S_{i-1}^{\prime}}^{-1}(t)\left|t_{n-i}^{\prime}\right|^{1-i} d \xi d t^{\prime} d t d y^{\prime}\right) d x^{\prime}
\end{aligned}
$$

with the inner integrals being absolutely convergent. Make a change of variable $y^{\prime} \mapsto\left(t^{\prime}\right)^{-1} y^{\prime} t^{\prime}$ and notice that $T_{i}^{0}$ acts trivially by conjugation on $\left(D_{r, i} \tilde{R}_{r, i}\right) \backslash R_{r}$. We get

$$
\Lambda(W)=\int_{\left(D_{r, i} \tilde{R}_{r, i}\right) \backslash R_{r}}\left(\int_{\left.\begin{array}{l}
{\left[D_{r, i}\right]} \\
f\left(t t^{\prime}\right) \delta_{\tilde{T}_{i-1}^{\prime}}^{-1}
\end{array} \int_{\tilde{T}_{i}^{0}} \int_{\tilde{D}_{i}} A_{i+1}(\pi)\left|t_{n-i}^{\prime}\right|^{1-i+r-n} d \xi d t^{\prime} d t d y^{\prime}\right) d x^{\prime} .}\right.
$$

Finally, note that $\tilde{D}_{i}=\tilde{D}_{r, i} \times{ }_{i} \tilde{D}_{r}$, and from (24),

$$
\delta_{S_{i-1}^{\prime}}^{-1}(t)\left|t_{n-i}^{\prime}\right|^{r+1-i-n}\left|t_{n-i+1}\right|^{r-i-1}=\delta_{S_{i}^{\prime}}^{-1}\left(t t^{\prime}\right) .
$$

Unwinding the integrals we obtain the Lemma.

REMARK 4.6. Since $f(t)$ is an arbitrary function in $C_{c}^{\infty}\left(\tilde{T}_{i}\right)$, from the Lemma we get the convergence of

$$
\int_{\left(D_{r, i} \tilde{R}_{r, i}\right) \backslash R_{r}}\left(\int_{D_{r, i}} \int_{i \tilde{D}_{r}} A_{i+1}(\pi(\xi y x) W) d \xi d y\right) d x .
$$

In particular we get:

$$
\begin{aligned}
\Lambda(W)= & \int_{\tilde{T}_{i}} \int_{\left(D_{r, i} \tilde{R}_{r, i}\right) \backslash R_{r}}\left(\int_{D_{r, i}} \int_{i \tilde{D}_{r}}\right. \\
& \left.A_{i+1}(\pi(\xi y x t) W) f(t) \delta_{S_{i}^{\prime}}^{-1}(t)\left|t_{n-i+1}\right|^{1+i-r} d \xi d y\right) d x d t .
\end{aligned}
$$


4.6. From (25) and (27), we get:

$$
\begin{array}{r}
\mathcal{J}_{i}^{\prime}\left(W, \widehat{\kappa_{*} f}\right)=\int_{\left[_{i} C_{r}^{\circ}\right]}\left(\int _ { \tilde { T } _ { i } } \int _ { ( D _ { r , i } \tilde { R } _ { r , i } ) \backslash R _ { r } } \left(\int_{D_{r, i}} \int_{i \tilde{D}_{r}}\right.\right. \\
\left.\left.A_{i+1}(\pi(\xi y x t v) W) f(t) \delta_{S_{i}^{\prime}}^{-1}(t)\left|t_{n-i+1}\right|^{1+i-r} d \xi d y\right) d x d t\right) d v .
\end{array}
$$

Note that ${ }_{i} C_{r}^{\circ}$ and $D_{r, i} \tilde{R}_{r, i}$ commute. Making a change of variable $x \mapsto\left(t v t^{-1}\right) x\left(t v^{-1} t^{-1}\right)$, the above is:

$$
\begin{gathered}
\mathcal{J}_{i}^{\prime}\left(W, \widehat{\kappa_{*} f}\right)=\int_{\left[_{i} C_{r}^{\circ}\right]}\left(\int _ { \tilde { T } _ { i } } \int _ { ( D _ { r , i } \tilde { R } _ { r , i } ) \backslash R _ { r } } \left(\int_{D_{r, i}} \int_{i \tilde{D}_{r}} A_{i+1}\left(\pi\left(\xi t v t^{-1} y x t\right) W\right)\right.\right. \\
\left.\left.f(t) \delta_{S_{i}^{\prime}}^{-1}(t)\left|t_{n-i+1}\right|^{1+i-r} d \xi d y\right) d x d t\right) d v .
\end{gathered}
$$

From (23), we get

$$
A_{i+1}\left(\pi\left(\xi t v t^{-1} y x t\right) W\right)=A_{i+1}(\pi(\xi y x t) W)\left\langle t v t^{-1}, \xi\right\rangle .
$$

Clearly $\xi$ commutes with $y$ and acts trivially by conjugation on $\left(D_{r, i} \tilde{R}_{r, i}\right) \backslash R_{r}$. Thus the above is:

$$
\begin{aligned}
\mathcal{J}_{i}^{\prime}\left(W, \widehat{\kappa_{*} f}\right)= & \int_{\left[_{i} C_{r}^{\circ}\right]}\left(\int _ { ( D _ { r , i } \tilde { R } _ { r , i } ) \backslash R _ { r } } \int _ { \tilde { T } _ { i } } \left(\int_{D_{r, i}} \int_{i \tilde{D}_{r}} A_{i+1}(\pi(y x \xi t) W)\right.\right. \\
& \left.\left.f(t) \delta_{S_{i}^{\prime}}^{-1}(t)\left|t_{n-i+1}\right|^{1+i-r}\left\langle t v t^{-1}, \xi\right\rangle d \xi d y\right) d t d x\right) d v .
\end{aligned}
$$

The same argument as in Lemma 3.8, using Remark 4.6 in place of Lemma 3.7, shows that the triple integral over $x, t$ and $v$ above is absolutely convergent. Thus we may integrate over $v$ first.

$$
\begin{array}{r}
\mathcal{J}_{i}^{\prime}\left(W, \widehat{\kappa_{*} f}\right)=\int_{\left(D_{r, i} \tilde{R}_{r, i}\right) \backslash R_{r}} \int_{\tilde{T}_{i}} \int_{\left[_{i} C_{r}^{\circ}\right]}\left(\int_{D_{r, i}} \int_{i \tilde{D}_{r}} A_{i+1}(\pi(y x \xi t) W)\right. \\
\left.f(t) \delta_{S_{i}^{\prime}}^{-1}(t)\left|t_{n-i+1}\right|^{1+i-r}\left\langle t v t^{-1}, \xi\right\rangle d \xi d y\right) d v d t d x .
\end{array}
$$

Change $v \mapsto t^{-1} v t$ :

$$
\begin{array}{r}
\mathcal{J}_{i}^{\prime}\left(W, \widehat{\kappa_{*} f}\right)=\int_{\left(D_{r, i} \tilde{R}_{r, i}\right) \backslash R_{r}} \int_{\tilde{T}_{i}} \int_{\left.{ }_{i} C_{r}^{\circ}\right]}\left(\int_{D_{r, i}} \int_{i \tilde{D}_{r}} A_{i+1}(\pi(y x \xi t) W)\right. \\
\left.f(t) \delta_{S_{i}^{\prime}}^{-1}(t)\langle v, \xi\rangle d \xi d y\right) d v d t d x .
\end{array}
$$

By Lemma 3.7, $A_{i+1}(\pi(y x \xi t) W)$ is a Schwartz function in $\xi$ and $y$ for fixed $x$ and $t$. By Fourier inversion we get

$$
\mathcal{J}_{i}^{\prime}\left(W, \widehat{\kappa_{*} f}\right)=\int_{\left(D_{r, i} \tilde{R}_{r, i}\right) \backslash R_{r}} \int_{\tilde{T}_{i}}\left(\int_{D_{r, i}} A_{i+1}(\pi(y x t) W) f(t) \delta_{S_{i}^{\prime}}^{-1}(t) d y\right) d t d x .
$$


If we could unwind this expression we would get $\mathcal{J}_{i}^{\prime}\left(W, \widehat{\kappa_{*} f}\right)=A_{r, i}^{\prime}\left(\pi\left(f \delta_{S_{i}^{\prime}}^{-1}\right) W\right)$ and complete the induction step of the proof of Proposition 4.4.

4.7. We are left to show:

Lemma 4.7. Fix $i=0, \ldots, r-1$. Assume that the iterated integral

$$
\int_{\left(D_{r, i} \tilde{R}_{r, i}\right) \backslash R_{r}}\left(\int_{D_{r, i}} A_{i+1}(\pi(y x) W) d y\right) d x
$$

converges for all $W \in \mathcal{W}(\pi, \psi)$. Then the integral

$$
\int_{\tilde{R}_{r, i+1} \backslash R_{r}} A_{i+1}(\pi(x) W) d x=\int_{\left(D_{r, i} \tilde{R}_{r, i} \backslash \backslash R_{r}\right.} \int_{D_{r, i}} A_{i+1}(\pi(y x) W) d y d x
$$

is absolutely convergent for all $W \in \mathcal{W}(\pi, \psi)$.

To prove the Lemma, we need another auxiliary result:

Lemma 4.8. Fix $j=1 \ldots, r-1$ and let $R^{\prime}$ be a subgroup of $G$ which commutes with $D_{r, j}$ and centralizes $C_{j}$ modulo ${ }_{j} C_{r}^{\circ}$. Let $R^{\prime \prime}$ be a subgroup of $R^{\prime}$ and let $A(W)$ be a linear form on $\mathcal{W}(\pi, \psi)$ satisfying $A(\pi(r v) W)=\psi(v) A(W)$ for all $r \in R^{\prime \prime}$ and $v \in C_{j}$. Assume that the iterated integral

$$
\int_{R^{\prime \prime} \backslash R^{\prime}}\left(\int_{D_{r, j}} A(\pi(y x) W) d y\right) d x
$$

converges for all $W \in \mathcal{W}(\pi, \psi)$. Then the integral

$$
\int_{R^{\prime \prime} \backslash R^{\prime}} A(\pi(x) W) d x
$$

is absolutely convergent for all $W \in \mathcal{W}(\pi, \psi)$.

Proof. Let $W=\pi(\varphi) W^{\prime}$ where $\varphi \in \mathcal{S}\left(C_{r, j}\right)$. Then

$$
\begin{aligned}
\int_{R^{\prime \prime} \backslash R^{\prime}}\left(\int_{D_{r, j}} A(\pi\right. & (y x) W) d y) d x \\
= & \int_{R^{\prime \prime} \backslash R^{\prime}}\left(\int_{D_{r, j}} \int_{C_{r, j}} A\left(\pi(y x v) W^{\prime}\right) \varphi(v) d v d y\right) d x .
\end{aligned}
$$


Since $x$ centralizes $C_{j}$ modulo ${ }_{j} C_{r}^{\circ}$ and $D_{r, j}$ commutes with ${ }_{j} C_{r}^{\circ}$ we get by a change of variable $v \mapsto x^{-1} v x$ that

$$
\begin{aligned}
\int_{R^{\prime \prime} \backslash R^{\prime}} & \left(\int_{D_{r, j}} \int_{C_{r, j}} A\left(\pi(y v x) W^{\prime}\right) \varphi(v) d v d y\right) d x \\
= & \int_{R^{\prime \prime} \backslash R^{\prime}}\left(\int_{D_{r, j}} \int_{C_{r, j}} A\left(\pi(y x) W^{\prime}\right)\langle v, y\rangle \varphi(v) d v d y\right) d x \\
= & \int_{R^{\prime \prime} \backslash R^{\prime}}\left(\int_{D_{r, j}} A\left(\pi(x y) W^{\prime}\right) \hat{\varphi}(y) d y\right) d x=\int_{R^{\prime \prime} \backslash R^{\prime}} A\left(\pi(x) \pi(\hat{\varphi}) W^{\prime}\right) d x
\end{aligned}
$$

The claim follows from the Dixmier-Malliavin theorem as $\varphi$ is an arbitrary Schwartz function.

Proof of Lemma 4.7. Fix $i$ and $r$ and assume that the conditions of the Lemma hold. Note that

$$
\left(D_{r, i} \tilde{R}_{r, i}\right) \backslash R_{r}=\left[R_{r, i+1}\right] \times \prod_{j=1}^{i-1} D_{r, j} .
$$

We can apply Lemma 4.8 iteratively for $j=1, \ldots, i-1$, where $R^{\prime}=$ $R_{r, i+1} \times \prod_{k=j+1}^{i-1} D_{r, j}, R^{\prime \prime}=\tilde{R}_{r, i+1}$ and $A(W)=\int_{D_{r, i}} A_{i+1}(\pi(x) W) d x$. We conclude that the iterated integrals

$$
\int_{\left[R_{r, i+1}\right]}\left(\int_{D_{r, i}} A_{i+1}(\pi(y x) W) d y\right) d x
$$

converge. Apply Lemma 4.8 once again for $j=i$ with $R^{\prime}=R_{r, i+1}$, $R^{\prime \prime}=\tilde{R}_{r, i+1}$ and $A(W)=A_{i+1}(W)$. From (5), the conditions of the Lemma are satisfied. We get that the integral

$$
\int_{\left[R_{r, i+1}\right]} A_{i+1}(\pi(x) W) d x
$$

is absolutely convergent for all $W \in \mathcal{W}(\pi, \psi)$. Note that $\tilde{R}_{r, i+1} \backslash R_{r}=$ $\left[R_{r, i+1}\right] \times \prod_{j=1}^{i} D_{r, j}$. From the above convergence, we can derive the claim in Lemma 4.7 using the Dixmier-Malliavin Theorem, (9) and the last claim of Lemma 2.3.

The proof of Proposition 4.4, and hence of Proposition 4.2, is now complete.

\section{The functional equation}

5.1. Finally, we prove Theorem 1.2 .

We continue to work under the Assumptions 2.8 and 2.10. 
Suppose first that $\pi \in \Pi_{\text {temp }}(G)$ is $\tilde{G}$-distinguished. Then by (4), Proposition 4.2 and Lemma 4.1 we have

$B_{r}\left(W^{\sharp}\right)=A_{r}^{\prime}\left(\pi\left(w_{n, r}^{*} w_{n}\right) W\right)=\mathcal{E}_{r}^{\prime}\left(\pi\left(w_{n, r}^{*} w_{n}\right) W\right)=\mathcal{E}_{r}^{\prime}\left(\pi\left(\hat{w}_{r}\right) W\right)=\mathcal{E}_{r-1}\left(W^{\sharp}\right)$.

Theorem 1.2 now follows from Propositions 3.5 in this case.

Now let $\pi \in \Pi_{\text {gen }}(G)$ be unitarizable and $\tilde{G}$-distinguished. Consider the inert case. Let $\tau$ be the Galois involution of $E / F$. By Assumption 2.10 we can write

$$
\pi=\pi_{1} \times \cdots \times \pi_{k} \times \sigma_{1}^{\vee}\left[-s_{1}\right] \times \cdots \times \sigma_{l}^{\vee}\left[-s_{l}\right] \times \sigma_{1}^{\tau}\left[s_{1}\right] \times \cdots \times \sigma_{l}^{\tau}\left[s_{l}\right]
$$

where $\pi_{1}, \ldots, \pi_{k}$ are square-integrable and distinguished, $\sigma_{1}, \ldots, \sigma_{l}$ are square-integrable and $-\frac{1}{2}<s_{1}, \ldots, s_{l}<\frac{1}{2}$ (since $\pi$ is unitarizable). Consider the Jacquet integral $J(\varphi, \underline{s}), \underline{s} \in \mathbb{C}^{l}$ with respect to the inducing representation. We then view the identity

$$
A_{r}(J(\varphi, \underline{s}))=B_{r}(J(\varphi, \underline{s}))
$$

as an identity of holomorphic functions in the strip $-\frac{1}{2}<\operatorname{Re} \underline{s}_{i}<\frac{1}{2}$. Since it holds for $\underline{s}_{i} \in \mathrm{i} \mathbb{R}$, it holds in the entire strip. Specializing to $\underline{s}=\left(s_{1}, \ldots, s_{l}\right)$ we get $A_{r}(W)=B_{r}(W)$. A similar argument works in the split case.

5.2. We can extend Theorem 1.2 (still under Assumptions 2.8 and 2.10) as follows.

THEOREM 5.1. Let $\pi \in \Pi_{\text {gen }}(G)$ be unitarizable and $\tilde{G}$-distinguished. Then for $W \in \mathcal{W}(\pi, \psi)$ and $1 \leq i \leq r \leq n-1$ we have

$$
\begin{aligned}
\int_{R_{r, i}} \int_{\tilde{N}_{r} \backslash \tilde{H}_{r}} W(h y)|\operatorname{det} h|^{1-r} d h d y & \\
=\int_{R_{r, r-i+1}^{*}} & \int_{\tilde{N}_{r}^{*} \backslash \tilde{H}_{r}^{*}} W\left(h x w_{n, r}\right)|\operatorname{det} h|^{r-1} d h d x .
\end{aligned}
$$

Proof. It follows from Lemma 2.3 and its proof that both sides of the equation are absolutely convergent. Once again, we can reduce to the tempered case by analytic continuation.

The equation for $i=r$ is just Theorem 1.2. Now assume that the equation holds for $i=l+1$, and we prove it for $i=l$. By induction 
hypothesis:

$$
\begin{aligned}
& \int_{R_{r, l}} \int_{\tilde{N}_{r} \backslash \tilde{H}_{r}} W(h y)|\operatorname{det} h|^{1-r} d h d y \\
= & \int_{D_{r, l}} \int_{R_{r, l+1}} \int_{\tilde{N}_{r} \backslash \tilde{H}_{r}} W(h u v)|\operatorname{det} h|^{1-r} d h d u d v \\
= & \int_{D_{r, k}}\left(\int_{R_{r, r-l}^{*}} \int_{\tilde{N}_{r}^{*} \backslash \tilde{H}_{r}^{*}} W\left(h x w_{n, r} v\right)|\operatorname{det} h|^{r-1} d h d x\right) d v \\
= & \int_{D_{r, l}}\left(\int_{D_{r, r-l}^{*}} \int_{R_{r, r-l+1}^{*}} \int_{\tilde{N}_{r}^{*} \backslash \tilde{H}_{r}^{*}} W\left(h x \xi w_{n, r} v\right)|\operatorname{det} h|^{r-1} d h d x d \xi\right) d v .
\end{aligned}
$$

As $W\left(h x \xi w_{n, r} v\right)=W\left(h x \xi w_{n, r}\right)\left\langle w_{n, r}^{-1} \xi w_{n, r}, v\right\rangle$, we get by Fourier inversion that the above is just

$$
\int_{R_{r, r-l+1}^{*}} \int_{\tilde{N}_{r}^{*} \backslash \tilde{H}_{r}^{*}} W\left(h x w_{n, r}\right)|\operatorname{det} h|^{r-1} d h d x
$$

as required.

\section{References}

[AG09] Avraham Aizenbud and Dmitry Gourevitch, Generalized HarishChandra descent, Gelfand pairs, and an Archimedean analog of JacquetRallis's theorem, Duke Math. J. 149 (2009), no. 3, 509-567, With an appendix by the authors and Eitan Sayag. MR 2553879 (2011c:22026)

[Bar03] Ehud Moshe Baruch, A proof of Kirillov's conjecture, Ann. of Math. (2) 158 (2003), no. 1, 207-252. MR 1999922 (2004f:22012)

[Ber84] Joseph N. Bernstein, P-invariant distributions on $\mathrm{GL}(N)$ and the classification of unitary representations of $\mathrm{GL}(N)$ (non-Archimedean case), Lie group representations, II (College Park, Md., 1982/1983), Lecture Notes in Math., vol. 1041, Springer, Berlin, 1984, pp. 50-102. MR 748505 (86b:22028)

[CHH88] M. Cowling, U. Haagerup, and R. Howe, Almost $L^{2}$ matrix coefficients, J. Reine Angew. Math. 387 (1988), 97-110. MR MR946351 (89i:22008)

[Fli88] Yuval Z. Flicker, Twisted tensors and Euler products, Bull. Soc. Math. France 116 (1988), no. 3, 295-313. MR 984899 (89m:11049)

[Fli91]_ On distinguished representations, J. Reine Angew. Math. 418 (1991), 139-172. MR 1111204 (92i:22019)

[FLO12] Brooke Feigon, Erez Lapid, and Omer Offen, On representations distinguished by unitary groups, Publ. Math. Inst. Hautes Études Sci. (2012), 185-323. MR 2930996

[Jac04] Hervé Jacquet, Integral representation of Whittaker functions, Contributions to automorphic forms, geometry, and number theory, Johns Hopkins Univ. Press, Baltimore, MD, 2004, pp. 373-419. MR 2058615 (2005f:11100) 
[Jac09] - Archimedean Rankin-Selberg integrals, Automorphic forms and $L$-functions II. Local aspects, Contemp. Math., vol. 489, Amer. Math. Soc., Providence, RI, 2009, pp. 57-172. MR 2533003 (2011a:11103)

[JPSS79a] Hervé Jacquet, Ilja Iosifovitch Piatetski-Shapiro, and Joseph Shalika, Automorphic forms on GL(3). I, Ann. of Math. (2) 109 (1979), no. 1, 169-212. MR 519356 (80i:10034a)

[JPSS79b] _ Automorphic forms on GL(3). II, Ann. of Math. (2) 109 (1979), no. 2, 213-258. MR 528964 (80i:10034b)

[JPSS83] H. Jacquet, I. I. Piatetskii-Shapiro, and J. A. Shalika, Rankin-Selberg convolutions, Amer. J. Math. 105 (1983), no. 2, 367-464. MR 701565 (85g:11044)

[JS81a] H. Jacquet and J. A. Shalika, On Euler products and the classification of automorphic forms. II, Amer. J. Math. 103 (1981), no. 4, 777-815. MR 623137 (82m:10050b)

[JS81b] On Euler products and the classification of automorphic representations. I, Amer. J. Math. 103 (1981), no. 3, 499-558. MR 618323 (82m:10050a)

[JS90] Hervé Jacquet and Joseph Shalika, Rankin-Selberg convolutions: Archimedean theory, Festschrift in honor of I. I. Piatetski-Shapiro on the occasion of his sixtieth birthday, Part I (Ramat Aviv, 1989), Israel Math. Conf. Proc., vol. 2, Weizmann, Jerusalem, 1990, pp. 125-207. MR 1159102 (93d:22022)

[KT08] Shin-ichi Kato and Keiji Takano, Subrepresentation theorem for p-adic symmetric spaces, Int. Math. Res. Not. IMRN (2008), no. 11, Art. ID rnn028, 40. MR 2428854 (2009i:22021)

[Lag08] Nathalie Lagier, Terme constant de fonctions sur un espace symétrique réductif p-adique, J. Funct. Anal. 254 (2008), no. 4, 1088-1145. MR MR2381204 (2009d:22013)

[LM] Erez Lapid and Zhengyu Mao, On Whittaker-Fourier coefficients of automorphic forms on $\widetilde{S p}_{2 n}$, preprint, Available at http://www.ma.huji. ac.il/ erezla/publications.html.

[LM09] - On the asymptotics of Whittaker functions, Represent. Theory 13 (2009), 63-81. MR 2495561 (2010b:22024)

[Mat11] Nadir Matringe, Distinguished generic representations of GL $(n)$ over $p$ adic fields, Int. Math. Res. Not. IMRN (2011), no. 1, 74-95. MR 2755483 (2012f:22032)

[Off11] Omer Offen, On local root numbers and distinction, J. Reine Angew. Math. 652 (2011), 165-205. MR 2787356

[Ran39] R. A. Rankin, Contributions to the theory of Ramanujan's function $\tau(n)$ and similar arithmetical functions. I. The zeros of the function $\sum_{n=1}^{\infty} \tau(n) / n^{s}$ on the line $\mathfrak{R} s=13 / 2$. II. The order of the Fourier coefficients of integral modular forms, Proc. Cambridge Philos. Soc. 35 (1939), 351-372. MR 0000411 (1,69d)

[Sel40] Atle Selberg, Bemerkungen über eine Dirichletsche Reihe, die mit der Theorie der Modulformen nahe verbunden ist, Arch. Math. Naturvid. 43 (1940), 47-50. MR MR0002626 (2,88a) 
[Shi76] Takuro Shintani, On an explicit formula for class-1 "Whittaker functions" on $G L_{n}$ over P-adic fields, Proc. Japan Acad. 52 (1976), no. 4, 180-182. MR MR0407208 (53 \#10991)

[Sun09] Binyong Sun, Bounding matrix coefficients for smooth vectors of tempered representations, Proc. Amer. Math. Soc. 137 (2009), no. 1, 353357. MR 2439460 (2010g:22023)

[SV] Yiannis Sakellaridis and Akshay Venkatesh, Periods and harmonic analysis on spherical varieties, preprint, arXiv:1203.0039v1.

[Wa192] Nolan R. Wallach, Real reductive groups. II, Pure and Applied Mathematics, vol. 132, Academic Press Inc., Boston, MA, 1992. MR 1170566 (93m:22018)

Department of Mathematics, The Weizmann Institute of Science, REHOVOT 76100, ISRAEL

E-mail address: erez.m.lapid@gmail.com

Department of Mathematics and Computer Science, Rutgers UniVERSITY, NEWARK, NJ 07102, USA

E-mail address: zmao@rutgers.edu 